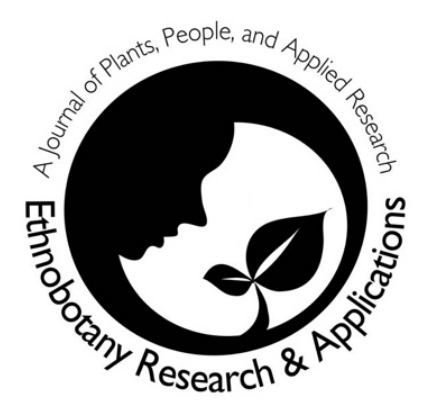

\title{
Ethnobotanical indigenous knowledge of Tehsil Charhoi, District Kotli, Azad Jammu and Kashmir, Pakistan
}

\author{
Usman Arif, K.H. Bhatti, M. Ajaib, Nasir Aziz Wagay, M. \\ Majeed, Jamal Zeb, Anmol Hameed and Jawad Kiani
}

\begin{abstract}
Correspondence
Usman Arif ${ }^{1}$, K.H. Bhatti ${ }^{1}$, M. Ajaib ${ }^{2}$, Nasir Aziz Wagay ${ }^{3}$, M. Majeed ${ }^{1}$, Jamal Zeb ${ }^{1}$, Anmol Hameed ${ }^{1}$ and Jawad Kiani $^{4}$

${ }^{1}$ Department of Botany, University of Gujrat, Gujrat, Pakistan

2Department of Botany, Mirpur University of Science and Technology (MUST), Mirpur, Azad Jammu and Kashmir, Pakistan

${ }^{3}$ Department of Botany, Government Degree College Baramulla, J\&K

${ }^{4}$ Department of Chemistry, Mohi-ud-Din Islamic University (MIU), Nerian Sharif, Azad Jammu and Kashmir, Pakistan

*Corresponding Authors: usmanbotany76@gmail.com
\end{abstract}

Ethnobotany Research \& Applications 22:50 (2021)

\section{Databases and Inventories}

\begin{abstract}
Background: Wild plants are used a lot by the local people of Azad Jammu and Kashmir (AJ\&K), Pakistan, for different purposes in daily life. It has been published by various researchers that AJ\&K, Pakistan is enriched with wild medicinal flora on the basis of surveys conducted in various parts but some areas are still unexplored.
\end{abstract}

Methods: The present study was conducted from March 2019 to April 2020 and 60 native informants were interviewed from different sites of Tehsil Charhoi. Ethnomedicinal data was collected using a semi-structured questionnaire.

Results: In ethno-botanical survey total 100 species of plant were documented that belonging to 52 families and 90 genera. Moraceae was the dominant family in the study area that included 7 species (13.46\%). In present ethnobotanical survey total 16 plant parts were utilized for preparation of traditional remedies against for various ailments. The most used plant parts were leaves (28\%), followed by roots (18\%), fruits (12\%), bark, seeds and whole plants ( $8 \%$ each), stems (4\%), flowers, wood and latex ( $3 \%$ each), and resin, rhizomes, pods, tubers, gum and aerial parts ( $1 \%$ each). We evidenced a total 18 kinds of preparations curing various diseases with paste (59\%) being the most common. The Use Value (UV) index ranged from 0.2 to 0.96 . Highest UV was reported for Cuscuta reflexa Roxb. (0.96) whereas lowest UV was reported for Rhamnus triquetra (Wall) Brandis. The Relative Frequency of Citation (RFC) values ranged 0.03 to 0.55 . The highest RFC value was obtained for Ficus benghalensis $\mathrm{L}$. (0.55) while Gloriosa superba L. (0.03) had the lowest value. The Informant Consensus Factor (ICF) values ranged 0.21 to 0.68. Diseases were classified into 17 different categories. The high ICF value recorded for jaundice and hepatitis whereas low ICF value for cardiac problems. The highest Fidelity Level (FL) value of Viola canescens Wall (97.1\%) was helpful for colds, flu, coughs, constipation, and jaundice. In present study out of 100 medicinal plant species 30 plant species were also utilized for other than medicinal purposes like vegetable, fruit, fodder, fuel, furniture, making agriculture tools, house thatching, walking sticks and handicrafts. In direct matrix ranking of multipurpose species (DMRMS) Dodonaea viscosa (L.) Jacq. $r$ nked first according to information provided by 10 informants. 
Conclusion: It has been concluded that Tehsil Charhoi, District Kotli, Azad Jammu and Kashmir are rich sources of wild plants used to treat various ailments. There is need to build awareness among local people about the value of medicinal plants and the publication of ethno-botanical information forms the basis for the development of innovative drugs.

Keywords: Charhoi, Indigenous, Ethnobotany, Wild, Kotli

\section{Background}

Green plants are the transducers and primary producers on this planet earth and all animals including human beings are dependent upon them. Medicinal plants are being used to treat almost all types of ailments from prehistoric times (Akinyemi et al. 2006, Wagay et al. 2014). Plants are utilized as primary medications to cure various diseases even in most of the rural areas of well-developed countries (Chitme et al. 2004). Most of the allopathic medicines are based on medicinal plants (Qureshi et al. 2007, Ajaib \& Khan 2014). Almost 2000 species of plants were utilized in Homeopathy, Ayurvedic and Unani systems of medicines (Kritikar \& Basu 2001). Residents of farflung areas are often dependent on ethnomedicinal knowledge for the cure of various diseases. Plants also provide food, shelter, fodder, firewood, and lumber (Abbas et al. 2020). Ethnobotanical knowledge preserved in the form of documentation with the efforts of the ethnobotanists before its extinction will be highly useful for future generations (Rao \& Henry 1997). An ethnobotanical study also aids in the identification of the aromatic and medicinally important plants, hence forming the basis for drug discovery. Allopathic drugs are mostly derived from the extracts of these ethno-medicinally important plants (Rashid \& Arshad 2002). Chemicals produced in plants contain wide range of medicinal properties (Wagh \& Jain 2020). Interactions between humans and plants have long been described as a major factor in human civilization, especially in the medical field (Yeung et al. 2020). Pakistan has different climatic, ecological zones and topography. The flora of Pakistan and Azad Jammu and Kashmir is very diverse nearly; there are various types of flowering and non-flowering plants present. It is reported that about 6000 wild flowering plant species are present in Pakistan and Azad Jammu and Kashmir among them 600 are most commonly utilized as good source of medicines (Khan et al. 2012). According to a report made by WHO, 65-80\% people of whole world utilized plant for primary health care (Calixto, 2005). In Pakistan about $80 \%$ people lives in rural areas and medicinal plants are readily available there (Ahmad et al. 2016). Plants are being used in treatment of various diseases and phytochemicals are bringing used in making of synthetic medicines (Aggarwal 2003). Folk knowledge related to use of plants transmitted orally from one generation to another (Malik \& Singh 2019). The traditional ethnobotanical knowledge held by hakims, old age people and passed to the younger generation with the help of verbal communication (Amiri \& Joharchi 2013). The literature on the usage of medicinal plant species in ethnobotanical studies empowers us for the formulation of modern medicines which are being used for the treatment of various diseases and for the preservation of these medicinal plants (Calzada $\&$ Bautista 2020).

Amjad et al. (2020) documented ethnobotanical uses of 150 plant species having medicinal values. Altaf et al. (2019) explored ethnomedicinal importance of 97 plants that were utilized as therapeutic agents in Tehsil Wazirabad, Gujranwala, Pakistan. Ajaib et al. (2016) recorded 100 plant species that were utilized to treat various ailments in Puna Hills, Tehsil Samahni, Azad Jammu and Kashmir. Sulaiman et al. (2020) investigated 109 traditional medicinal plants of Gokand, District Buner, Pakistan. Ahmadet al. (2012) studied floristic and ethnobotanical research in Senhsa, District Kotli, Azad Jammu and Kashmir, recorded total 112 plant species that belong to 97 genera and 51 families having medicinal importance. Ajaib et al. (2015) investigated the ethnobotanical examination of the medicinal plants of Village Darguti, Tehsil Khuiratta, District Kotli of Azad Jammu and Kashmir, total 100 species of plants were found that were belonging to 47 families having ethnobotanical values. Ajaib and Khan (2014) explored ethnobotanically important trees of District Kotli, Azad Jammu and Kashmir, total 50 species of trees were recorded, related to 49 genera and 24 families. In dicot Moraceae was a very common family having 9 species of plants. We found only one gymnosperm family Pinaceae whereas all other angiosperm families, 2 monocot and 47 dicot tree species having medicinal values. Mahmood et al. (2011) conducted ethnomedicinal research in the Bhimber District of Azad Kashmir and a total of 48 plant species of 22 families were recorded using for ethnomedicinal purposes. Saghir et al. (2001) investigated ethno-botanical knowledge of Chikar and kindred areas of Muzaffarabad, Azad Jammu, and Kashmir, total 54 plant species were recorded in his study that belonged to 48 genera of 44 families having ethnobotanical importance. Ishtiaq et al. (2021) explored 150 plant species that belonging to 58 families having ethnobotanical importance.

According to above mentioned importance and information about medicinal plants, this study helps to records ethno-botanical knowledge of Tehsil Charhoi, District Kotli, Azad Jammu and Kashmir where such work has not 
been done up to now and to protect the threatened species and record the ethno-botanical data of those threatened species. This study also helps to create attentiveness among indigenous people about the usage and conservation of ethnobotanically important plant species.

\section{Materials and Methods}

\section{Study Area Description}

Azad Jammu and Kashmir is region of Kashmir under the control of Pakistan. It is also known as Pakistan Occupied Kashmir (POK) having total area of 13,297 Sq. Kilometers and is situated in the North-East of Pakistan and NorthWest of India. The Azad Jammu and Kashmir consists of 10 Districts and 32 Tehsils. This state is rich with natural resources and most of the area is hilly. The highest peak of state is Sarwali (6326 m) located in Neelum valley.

The present study was conducted during 2019-2020 in different sites of Tehsil Charhoi, District Kotli, Azad Jammu \&Kashmir. The present survey was conducted in Charhoi, Kotli Sohlan, Panjan, Narmah, Dahmal, Kotehra Khanka, Narakot, Saneh Baneh, Narmah, Palahal and Kaladab. Geographically present study area located near latitude $44^{\circ}$ 19'44.42" N and longitude $74^{\circ} 1$ '21" E (Figure 1).

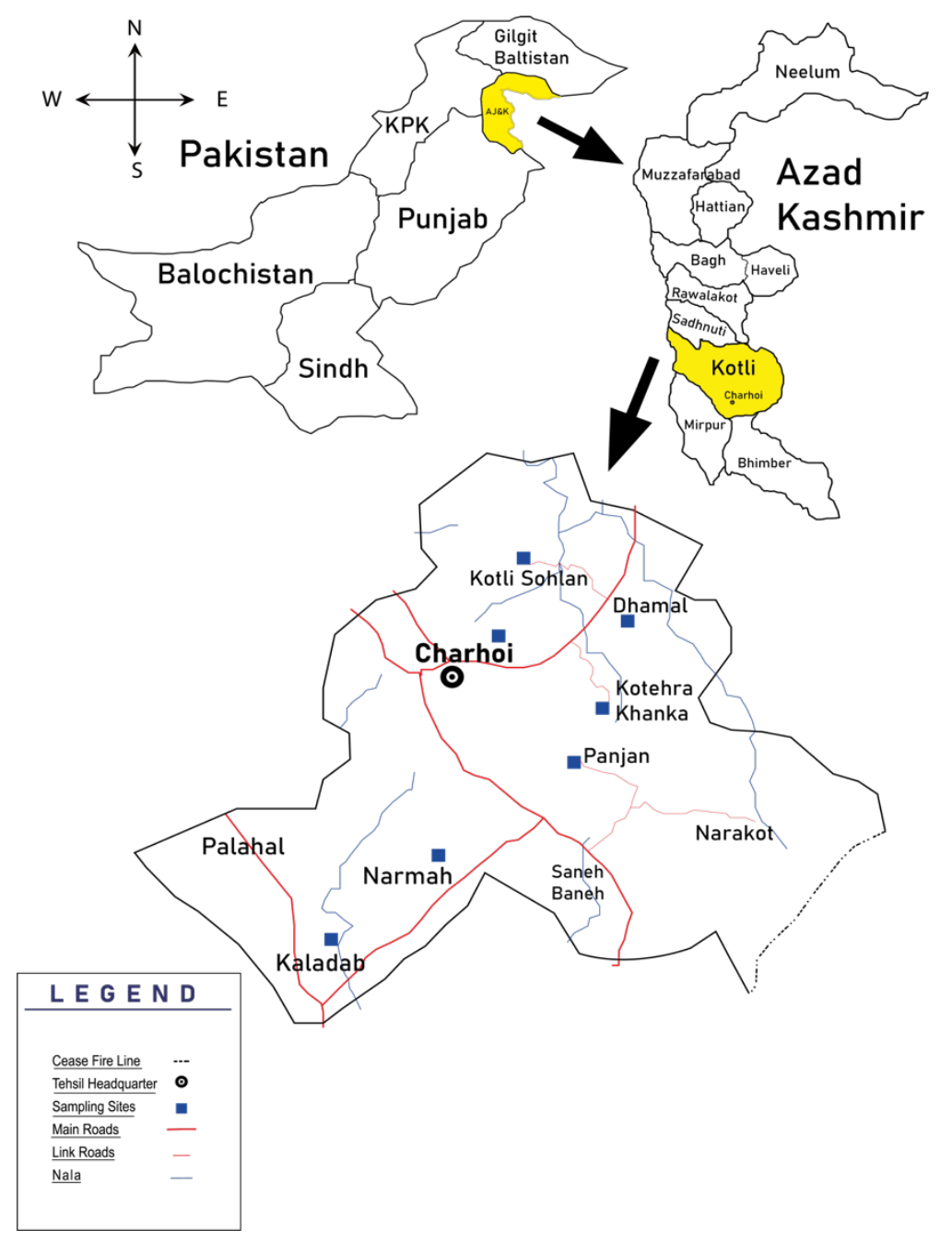

Figure 1. Map of Study area Tehsil Chahoi, District Kotli, Azad Jammu and Kashmir

Charhoi is Tehsil in Kotli District and is one of the most beautiful valleys in Kashmir. The valley of Charhoi is surrounded by high mountains all around. The mountains are covered with Cheer (Pine) plants (Pinus roxburghii Sarg.) that increase the beauty of this area. It is 64 kilometers from Mirpur and about 40 kilometers from khuiratta. Narakot, Sanyah, Kaladab, chahwala, Dhamal Bazar, Khanka Kotera, Panjni, are the main places of this area. The temperature of this area varies according to season. The June and July are warmest months of the year. In June the average temperature is $41^{\circ} \mathrm{C}$. January is the coldest month, with temperatures averaging $10.1^{\circ} \mathrm{C}$. It is bounded by 
Indian Occupied Kashmir (Cease Fire Line) in the East, in the south Union Council Poona of Tehsil Samahni, in the North District Kotli, Tehsil Khuiratta of District Kotli is between the East and North, and in the west Union Council Islamgarh of Tehsil Chakswari. The area varies in climate from subtropical type to humid with an average rainfall of about $92.5 \mathrm{~mm}$ per month. The average altitude of this area is $840 \mathrm{~m}$ and it lies in a sub-tropical zone (Anonymous 2020).

\section{Material description}

The present study area visited after regular intervals to collect ethnobotanical data. Plant specimens were collected from natural habitats, including complete vegetative and floral characters and took pictures of the habitat and plant that helped in identification. Collected plants were dried, pressed, stored, and finally placed on standard herbarium sheets according to the technique as described by Miller and Nyberg (1995). Field notebook, pencil, cutter, newspapers, plant presser, polythene bags, digging tools and digital camera were used for collection of information.

\section{Collection of data from field}

Ethnomedicinal data was collected using a systematic questionnaire. The local inhabitants were interviewed to get data about the use of plants for medicine, food, fuel, fodder, and shelter etc. All ethnomedicinally important plant species were arranged with names of families, names of species, common names and parts utilized. During investigation, data was collected from native people of Tehsil Charhoi, District Kotli, Azad Jammu \& Kashmir. Mostly data was collected from old age people that were familiar about the usage of plants. Most people of this area easily understand Kashmiri language so during the questionnaire this language was used. A new generation of locals who are unaware of the importance of herbal medicines and ethnobotanical knowledge is limited to the elderly. After old age people ethnobotanical knowledge of this area may be extinct so documentation is needed to preserve the data for future generations. The ethnomedicinal information was primarily collected from Herbal practitioners (Hakeems) of this area and additionally information was harvested from housewives. The collected specimens were identified with the help of available floristic literature (Ahmad et al. 2012, Amjad 2015, Ajaib \& Khan 2014, Amjad et al. 2017, Amjad \& Arshad 2014) and Flora of Pakistan and the collected specimens were classified in accordance of Bentham \& Hooker classification. Later the doubtful plant specimens were confirmed by deposited herbarium specimens at the Department of Botany Herbarium, University of Gujrat Pakistan. The Plant List (www.theplantlist.org) was used to obtain the correct nomenclature of collected species of study area. The plant specimens were submitted to Herbarium, Department of Botany, University of Gujrat, Gujrat Pakistan after pasting voucher numbers.

\section{Analysis of data}

The ethno-botanical data was evaluated with the help of different quantitative indices to test the similarity and validation, such as Use value (UV), Relative frequency of citation (RFC), Informant consensus factor (ICF), Fidelity level (FL), DMR and Family Index (FI).

\section{Use Value (UV)}

The relative importance of given species was collected from the area determined by Use Value (UV). The Use Value (UV) was calculated with the help of a formula extracted from previous paper of Umair et al. (2017).

$U V=\Sigma U i / N$

Where, $\mathrm{Ui}=$ Informant mentioned number of uses of a given species. $\mathrm{N}=$ Total number of informants.

\section{Relative frequency of citation (RFC)}

It indicates the medicinal importance of plants of specific area which is measured in the form of relative frequency of citation (RFC) by following the protocol of Umair et al. (2017).

$\mathrm{RFC}=\mathrm{FC} / \mathrm{N}$

Where, FC is the informants reporting the number of uses of a species. $\mathrm{N}=$ is the total number of informants.

\section{Fidelity level (FL)}

The fidelity level (FL) of the records, illustrates percentage of informers demanding the any use of a specific plant for the similar main purpose or ailment group and compared with entire described practices of all species of plant 
to treat the similar group of ailments. Its formula is as under and extracted from previous work of Amjad et al. (2017).

$\mathrm{FL}(\%)=(\mathrm{Np} / \mathrm{N}) \times 100$

Where, $\mathrm{Np}$ is the number of people who claim to use the plant species for a disease. $\mathrm{N}=$ is the number of people who use plants for any kind of disease.

\section{Informant consensus factor (ICF)}

Informant consensus factor (ICF) was determined for every group of ailments to recognize compromise of informers on the described ethno-medicinal usages for every group and ICF was calculated by following formula of Giday et al. (2009).

Nur - Nt / (Nur - 1)

Where, Nur is the number of use reports for any disease category. $\mathrm{Nt}=$ is the number of species used.

\section{Direct matrix ranking (DMR)}

Data matrix ranking (DMR) analysis was measured to find the several usages of given plants. DMR analysis describes multiple usages of one plant for various purposes as told by the local communities. Plant species used for several dedications other than medicinal use. For example, food, vegetables, fodder, fuel, and shelter (Ishtiaq et al. 2013).

\section{Family index}

Family Index (FI) with maximum number of plant species used belonging to a specific family was calculated from collected records and designated in graphic approach. The ranking of plant families is based on the number of species (Rahman et al. 2020).

\section{Results and Discussion}

\section{Documentation of ethno-botanical flora}

The present research work was conducted during 2019-2020 to collect ethno-botanical knowledge of Tehsil Charhoi, District Kotli, Azad Jammu and Kashmir. During a field survey total 100 plant species were collected that belong to 52 families and 90 genera. Similarly, Ajaib et al. (2015) studied 100 plant species of Darguti, Tehsil Khuiratta, Azad Jammu and Kashmir. Shabir et al. (2017) investigated 98 plant species of Devi Gali, District Poonch, Azad Jammu and Kashmir. The most dominant family was Moraceae in the study area including 7 species (13.46\%). According to previous research of Ajaib and Khan (2014) Moraceae was dominant family in District Kotli that including 9 species. The second most dominant family in the study area was Euphorbiaceae that including 6 species (11.53\%). Solanaceae and Asteraceae includes 5 species in each (9.61\%), Amaranthaceae and Lamiaceae included 4 species in each (9.61\%), Brassicaceae, Mimosaceae, Papilionaceae, Rhamnaceae and Apocynaceae included 3 species in each (5.76\%), Acanthaceae, Asclepiadaceae, Caesalpiniaceae, Malvaceae, Menispermaceae, Poaceae, Pteridaceae and Tiliaceae includes 2 species in each (3.84\%), while remaining families includes 1 species in each (Figure 2). The present ethno-botanical flora was including only 1 Gymnosperm, 2 Pteridophytes and 97 Angiosperms (Ajaib \& Khan 2014). Details on all species are given in Table 1.

\section{Demographic data of Informants}

In present survey total 60 native informants were selected for ethno-botanical data collection. Among them, 30 men (50\%), 20 women (33.33\%) and 10 herbal practitioners (16.16\%) were interviewed to collect ethno-botanical data. The informants were belonging to 3 main age groups (i) 20 to 40 , (ii) 40 to 60 and (iii) 60 to 80 years old. Most of the informants were between age group 60-80 (30), 20 between age group 40-60 and 10 between age group 20-40 year (Figure 3). Similarly, Tesfaye et al. (2020) investigated traditional medicinal plants used in Ethopia. In this survey 48 herbal practitioners ( 37 men and 5 women) of different age groups (30 to 80 years) were randomly selected for the collection of ethnobotanical knowledge. Data collected by semi-structured interview or openended questions, information related to plants were collected and write on field notebook. Most of ethno-botanical was collected from old age peoples because old age peoples were more familiar about use of medicinal plants as compared to young generation. The traditional ethnobotanical knowledge held by hakims, old age people and passed to the younger generation with the help of verbal communication (Amiri and Joharchi, 2013). 
Table 1. Plant names, family names, mode of preparation and uses

\begin{tabular}{|c|c|c|c|c|c|c|c|c|c|}
\hline Family name & Botanical name & $\begin{array}{l}\text { Common } \\
\text { name }\end{array}$ & Habit & $\begin{array}{l}\text { Cultivated } \\
\text { status }\end{array}$ & Part used & $\begin{array}{l}\text { Mode of } \\
\text { preparation }\end{array}$ & UV & RFC & Uses \\
\hline Amaranthaceae & $\begin{array}{l}\text { Achyranthes aspera L. } \\
\text { UOG-788 }\end{array}$ & Puth Kanda & $\mathrm{H}$ & Wild & $\begin{array}{l}\text { Whole plant, } \\
\text { Ash, Leaves, } \\
\text { Roots }\end{array}$ & $\begin{array}{l}\text { Decoction, } \\
\text { Powder, Ash }\end{array}$ & 0.91 & 0.53 & $\begin{array}{l}\text { Rheumatic, diabetic, cough, asthma, skin } \\
\text { irritation, joints pains, piles, boil, abscess, } \\
\text { stomachic, digestant, pneumonia, toothache, } \\
\text { anti-fertility. }\end{array}$ \\
\hline Fabaceae & $\begin{array}{l}\text { Acacia modesta Wall. } \\
\text { UOG-790 }\end{array}$ & Phalai & T & Wild & $\begin{array}{l}\text { Wood, } \\
\text { Leaves, } \\
\text { Resin }\end{array}$ & Paste & 0.74 & 0.43 & Waist pain, clean teeth, tonic and stimulant. \\
\hline Pteridaceae & $\begin{array}{l}\text { Adiantum capillus-veneris } \mathrm{L} . \\
\text { UOG-791 }\end{array}$ & $\begin{array}{l}\text { Maidenhair } \\
\text { fern }\end{array}$ & $\mathrm{H}$ & Wild & Fronds & Tea, Paste & 0.36 & 0.18 & $\begin{array}{l}\text { Antidandruff, astringent, febrifuge, cough, } \\
\text { bronchitis, snakebites, headache, chest pains. }\end{array}$ \\
\hline Pteridaceae & $\begin{array}{l}\text { Adiantum incisum Forssk. } \\
\text { UOG-792 }\end{array}$ & Putramchari & $\mathrm{H}$ & Wild & Fronds & Paste, Juice & 0.33 & 0.16 & Cough, diabetes, skin diseases, fever. \\
\hline Amaranthaceae & $\begin{array}{l}\text { Aerva javanica (Burm. f.) Juss. } \\
\text { ex J.A. Schultes } \\
\text { UOG-793 }\end{array}$ & Boi & $S$ & Wild & $\begin{array}{l}\text { Leaves and } \\
\text { roots }\end{array}$ & $\begin{array}{l}\text { Paste, } \\
\text { Decoction, } \\
\text { Extract }\end{array}$ & 0.53 & 0.13 & $\begin{array}{l}\text { Pimples, kidney disorders, rheumatism, } \\
\text { toothache, eye problems. }\end{array}$ \\
\hline Lamiaceae & $\begin{array}{l}\text { Ajuga integrifolia Buch.- } \\
\text { Ham. } \\
\text { UOG-794 }\end{array}$ & Kori buti & $\mathrm{H}$ & Wild & $\begin{array}{l}\text { Leaves, } \\
\text { Roots }\end{array}$ & $\begin{array}{l}\text { Juice, Extract, } \\
\text { Powder }\end{array}$ & 0.57 & 0.33 & $\begin{array}{l}\text { Stomachache, blood purifier, abdominal pain, } \\
\text { malaria, jaundice, mouth ulcer, burns, boils, stop } \\
\text { bleeding, inflammation, dysentery, diarrhea. }\end{array}$ \\
\hline Fabaceae & $\begin{array}{l}\text { Albizzia lebbeck (L.) Bth. } \\
\text { UOG-795 }\end{array}$ & Shirin & $T$ & Wild & $\begin{array}{l}\text { Seeds, } \\
\text { Leaves, Bark }\end{array}$ & $\begin{array}{l}\text { Powder, } \\
\text { Smoke, Extract }\end{array}$ & 0.62 & 0.36 & $\begin{array}{l}\text { Headache, Kehn, stomach pain, antiseptic, } \\
\text { cough, pile, diarrhea, eye problems, boil, } \\
\text { diabetes. }\end{array}$ \\
\hline Amaryllidaceae & $\begin{array}{l}\text { Allium jacquemontii Kunth } \\
\text { UOG-796 }\end{array}$ & Jangli Piyaz & $\mathrm{H}$ & Wild & Bulb & Extract & 0.66 & 0.33 & $\begin{array}{l}\text { Blood cholesterol level, digestive system, } \\
\text { circulatory system. }\end{array}$ \\
\hline Amaranthaceae & $\begin{array}{l}\text { Amaranthus viridis } \mathrm{L} . \\
\text { UOG-797 }\end{array}$ & Gunahr & $\mathrm{H}$ & Wild & $\begin{array}{l}\text { Leaves, } \\
\text { Seeds }\end{array}$ & $\begin{array}{l}\text { Extract, } \\
\text { Infusion, Sap }\end{array}$ & 0.73 & 0.36 & $\begin{array}{l}\text { Constipation, anti-snake bite, laxative, backache, } \\
\text { purify blood, eye infection. }\end{array}$ \\
\hline Apiacceae & $\begin{array}{l}\text { Anethum graveolens L. } \\
\text { UOG-798 }\end{array}$ & Soya & $\mathrm{H}$ & Cultivated & Whole plant & $\begin{array}{l}\text { Powder, Raw } \\
\text { seeds }\end{array}$ & 0.6 & 0.3 & $\begin{array}{l}\text { Increase milk production in females, useful in } \\
\text { digestion. }\end{array}$ \\
\hline Acanthaceae & $\begin{array}{l}\text { Barleria cristata L. } \\
\text { UOG-799 }\end{array}$ & $\begin{array}{l}\text { Kali } \\
\text { Barenker }\end{array}$ & $S$ & Wild & Leaves, Root & $\begin{array}{l}\text { Juice, } \\
\text { Decoction, } \\
\text { Paste }\end{array}$ & 0.63 & 0.31 & $\begin{array}{l}\text { Anemia, tooth pain, inflammation, wound, snake } \\
\text { bite, fever, diabetes, tuberculosis, cold, flu, } \\
\text { diaphoretic, antibacterial and expectorant. }\end{array}$ \\
\hline Bombaceae & $\begin{array}{l}\text { Bombax ceiba L. } \\
\text { UOG-800 }\end{array}$ & Simbal & $\bar{T}$ & Wild & $\begin{array}{l}\text { Wood, Bark, } \\
\text { Fruit, Root }\end{array}$ & $\begin{array}{l}\text { Paste, Powder, } \\
\text { Juice }\end{array}$ & 0.8 & 0.46 & $\begin{array}{l}\text { Body weakness, increase muscle strength, } \\
\text { pimples, skin lesions, sore joins, diarrhea, } \\
\text { constipation, piles, gynaecological disorder, } \\
\text { stomach ache, kidney and bladder ulcer. }\end{array}$ \\
\hline Fabaceae & $\begin{array}{l}\text { Bauhinia variegata } \mathrm{L} \text {. } \\
\text { UOG-801 }\end{array}$ & Kalyar & $T$ & Wild & $\begin{array}{l}\text { Flowers, } \\
\text { Floral buds, }\end{array}$ & $\begin{array}{l}\text { Juice, } \\
\text { Decoction, } \\
\text { Paste }\end{array}$ & 0.86 & 0.43 & $\begin{array}{l}\text { Diarrhea, dysentery, wound, skin disorders, } \\
\text { ulcers, worms, dyspepsia, piles, regularize }\end{array}$ \\
\hline
\end{tabular}


Ethnobotany Research and Applications

\begin{tabular}{|c|c|c|c|c|c|c|c|c|c|}
\hline & & & & & $\begin{array}{l}\text { Leaves, Bark, } \\
\text { Roots }\end{array}$ & & & & $\begin{array}{l}\text { menstrual cycle, carminative, obesity, laxative, } \\
\text { anthelmintics, tonic. }\end{array}$ \\
\hline Brassicaceae & $\begin{array}{l}\text { Brassica campestris L. } \\
\text { UOG-802 }\end{array}$ & Sarson & $\mathrm{H}$ & Cultivated & $\begin{array}{l}\text { Leaves, } \\
\text { Whole plant, } \\
\text { Seed oil }\end{array}$ & $\begin{array}{l}\text { Decoction, } \\
\text { Powder, Oil }\end{array}$ & 0.7 & 0.23 & $\begin{array}{l}\text { Constipation, leucorrhoea, menstrual disorder, } \\
\text { body weakness, antimicrobial and anti-lice. }\end{array}$ \\
\hline Nyctaginaceae & $\begin{array}{l}\text { Boerhavia diffusa L. } \\
\text { UOG- } 803\end{array}$ & Tangri & $\mathrm{H}$ & Wild & $\begin{array}{l}\text { Roots, } \\
\text { Whole plant }\end{array}$ & $\begin{array}{l}\text { Powder, } \\
\text { Decoction, } \\
\text { Paste, Juice }\end{array}$ & 0.55 & 0.41 & $\begin{array}{l}\text { Jaundice, ulcers, eye disorders, dysentery, } \\
\text { arthritis, respiratory diseases, diabetes, } \\
\text { inflammation, anemia, gonorrhea. }\end{array}$ \\
\hline Papilionaceae & $\begin{array}{l}\text { Butea monosperma Taub. } \\
\text { UOG-804 }\end{array}$ & Chachra & $\bar{T}$ & Wild & $\begin{array}{l}\text { Leaves,Flowe } \\
\text { r, Seeds, } \\
\text { Gum }\end{array}$ & $\begin{array}{l}\text { Juice, } \\
\text { Decoction }\end{array}$ & 0.9 & 0.45 & $\begin{array}{l}\text { Diarrhea, dysentery, ulcers, swellings, ringworm, } \\
\text { cold, cough, boils, pimples, wounds, leucorrhoea, } \\
\text { control bleeding, abortion pain, wormicide. }\end{array}$ \\
\hline Buddlejaceae & $\begin{array}{l}\text { Buddleja asiatica Lour. } \\
\text { UOG-805 }\end{array}$ & & $S$ & Wild & $\begin{array}{l}\text { Leaves, } \\
\text { Wood, Bark, } \\
\text { Root, Whole } \\
\text { plant }\end{array}$ & $\begin{array}{l}\text { Juice, } \\
\text { Decoction, } \\
\text { Paste }\end{array}$ & 0.53 & 0.13 & $\begin{array}{l}\text { Skin diseases, malaria, reduce fever, premature } \\
\text { abortion, tumors. }\end{array}$ \\
\hline Asclepiadaceae & $\begin{array}{l}\text { Calotropis procera (Aiton) } \\
\text { Dryand. } \\
\text { UOG-806 }\end{array}$ & Akk & $\mathrm{S}$ & Wild & $\begin{array}{l}\text { Root, Flower, } \\
\text { Latex, Bark }\end{array}$ & $\begin{array}{l}\text { Paste, Powder, } \\
\text { Juice }\end{array}$ & 0.86 & 0.43 & $\begin{array}{l}\text { Earache, arthritis, skin diseases, snake bites, } \\
\text { leprosy, elephantiasis, cold, cough, asthma. }\end{array}$ \\
\hline Cannabaceae & $\begin{array}{l}\text { Cannabis sativa } \mathrm{L} . \\
\text { UOG- } 807\end{array}$ & Bhang & $\mathrm{H}$ & $\begin{array}{l}\text { Cultivated/ } \\
\text { Wild }\end{array}$ & $\begin{array}{l}\text { Flowers, } \\
\text { Leaves, } \\
\text { Fibers }\end{array}$ & Powder & 0.8 & 0.33 & $\begin{array}{l}\text { Asthma, tetanus, hemorrhages, gonorrhea, } \\
\text { nervous disorders, narcotics, painkiller, nausea, } \\
\text { vomiting. }\end{array}$ \\
\hline Apocynaceae & $\begin{array}{l}\text { Carissa spinarum L. } \\
\text { UOG- } 808\end{array}$ & Granda & $\mathrm{S}$ & Wild & $\begin{array}{l}\text { Fruit, Leaves, } \\
\text { Roots }\end{array}$ & $\begin{array}{l}\text { Decoction, } \\
\text { Powder, } \\
\text { Extract }\end{array}$ & 0.85 & 0.28 & $\begin{array}{l}\text { Asthma, jaundice, kidney pain, cardiac problems, } \\
\text { stimulant, urine blockage, urinary bladder, } \\
\text { remittent fever, liver disorders, blood } \\
\text { deficiencies, wounds, sores, stomachic, purgative }\end{array}$ \\
\hline Caricaceae & $\begin{array}{l}\text { Carica papaya L. } \\
\text { UOG-809 }\end{array}$ & Popita & $T$ & Cultivated & $\begin{array}{l}\text { Leaves, } \\
\text { Fruits, Seeds }\end{array}$ & $\begin{array}{l}\text { Juice, Extract, } \\
\text { Paste, } \\
\text { Decoction }\end{array}$ & 0.76 & 0.31 & $\begin{array}{l}\text { Dengue fever, digestion, diarrhea, high blood } \\
\text { pressure, diabetes, hypertension, warts, wounds, } \\
\text { dysentery, vermifuge. }\end{array}$ \\
\hline Fabaceae & $\begin{array}{l}\text { Cassia fistula L. } \\
\text { UOG- } 810\end{array}$ & Amaltas & $T$ & Wild & $\begin{array}{l}\text { Pods, } \\
\text { Leaves, Bark, } \\
\text { Seeds }\end{array}$ & Pulp, Juice & 0.66 & 0.16 & $\begin{array}{l}\text { Abdomen pain, habitual constipation, purgative, } \\
\text { laxative, malaria, diabetes, blood poisoning, skin } \\
\text { problems. }\end{array}$ \\
\hline Apocynaceae & $\begin{array}{l}\text { Catharanthus roseus }(\mathrm{L} .) \mathrm{G} . \\
\text { Don. } \\
\text { UOG-811 }\end{array}$ & Sada bahar & $\mathrm{H}$ & Ornamental & $\begin{array}{l}\text { Leaves, } \\
\text { Flower, Root, } \\
\text { Stem }\end{array}$ & $\begin{array}{l}\text { Decoction, } \\
\text { Juice, Extract }\end{array}$ & 0.84 & 0.35 & $\begin{array}{l}\text { Diabetes, wounds, dysmenorrhea, piles, nasal } \\
\text { and mouth bleeding, high blood pressure, } \\
\text { irregular menstruation, dysentery, diarrhea, } \\
\text { anticancer. }\end{array}$ \\
\hline Vitaceae & $\begin{array}{l}\text { Cayratia trifolia (L.) Doman } \\
\text { UOG-812 }\end{array}$ & Amalbel & $\mathrm{S}$ & Wild & $\begin{array}{l}\text { Fruit, Leaves, } \\
\text { Roots }\end{array}$ & $\begin{array}{l}\text { Decoction, } \\
\text { Paste, Juice, } \\
\text { Infusion }\end{array}$ & 0.7 & 0.23 & $\begin{array}{l}\text { Snake bite, fever, boils, aphrodisiac, scurvy, } \\
\text { muscular pain, itch, dandruff, inflammation, } \\
\text { diabetes, ulcer, blood purifier, carminative, } \\
\text { expectorant. }\end{array}$ \\
\hline
\end{tabular}


Ethnobotany Research and Applications

\begin{tabular}{|c|c|c|c|c|c|c|c|c|c|}
\hline Chenopodiaceae & $\begin{array}{l}\text { Chenopodium album } \mathrm{L} \text {. } \\
\text { UOG-813 }\end{array}$ & Bathu & $\mathrm{H}$ & Wild & $\begin{array}{l}\text { Leaves, } \\
\text { Seeds, } \\
\text { Roots, Stems }\end{array}$ & $\begin{array}{l}\text { Poultice, Juice, } \\
\text { Tea, Paste }\end{array}$ & 0.8 & 0.33 & $\begin{array}{l}\text { Bug bites, rheumatic joints, feet swelling, urinary } \\
\text { problems, sunburn, bloody dysentery, } \\
\text { stomachache, diarrhea, burns, constipation. }\end{array}$ \\
\hline Asteraceae & $\begin{array}{l}\text { Cirsium arvense (L.) Scopoli. } \\
\text { UOG-814 }\end{array}$ & Leh & $\mathrm{H}$ & Wild & Root, Leaves & $\begin{array}{l}\text { Paste, } \\
\text { Decoction, } \\
\text { Chewing }\end{array}$ & 0.46 & 0.11 & $\begin{array}{l}\text { Toothache, astringent, tonic, antiphlogistic, } \\
\text { hepatic, worms, antiplogistic, indigestion. }\end{array}$ \\
\hline Menispermaceae & $\begin{array}{l}\text { Cissampelos pareira L. } \\
\text { UOG-815 }\end{array}$ & Battal Bel & $\mathrm{H}$ & Wild & $\begin{array}{l}\text { Root, } \\
\text { Leaves, } \\
\text { Rhizome }\end{array}$ & $\begin{array}{l}\text { Decoction, } \\
\text { Paste, Juice, } \\
\text { Infusion }\end{array}$ & 0.82 & 0.48 & $\begin{array}{l}\text { Relief tooth pain, leucorrhoea, snake bite, } \\
\text { carminative, astringent, diuretic, expectorant, } \\
\text { sedative, febrifuge, oblivision, gastrointestinal } \\
\text { problems, fever, cold, typhoid fever, anti- } \\
\text { conjunctivitis. }\end{array}$ \\
\hline Labiatae & $\begin{array}{l}\text { Colebrookea oppositifolia } \\
\text { Smith. } \\
\text { UOG-816 }\end{array}$ & Chitti Suhali & $S$ & Wild & Leaves, Root & $\begin{array}{l}\text { Juice, Poultice, } \\
\text { Decoction }\end{array}$ & 0.4 & 0.06 & $\begin{array}{l}\text { Wounds, bruises, fever, headache, dysentery, } \\
\text { epilepsy, ulcers. }\end{array}$ \\
\hline Commelinaceae & $\begin{array}{l}\text { Commelina benghalensis L. } \\
\text { UOG-817 }\end{array}$ & Chura & $\mathrm{H}$ & Wild & Leaves, Root & $\begin{array}{l}\text { Decoction, } \\
\text { Juice, Sap, } \\
\text { Poultice }\end{array}$ & 0.72 & 0.3 & $\begin{array}{l}\text { Skin swelling, leprosy, laxative, infertility, } \\
\text { stomach problems, eye ailments, burns and sore } \\
\text { throat. }\end{array}$ \\
\hline Malvaceae & $\begin{array}{l}\text { Corchorus aestuans } \mathrm{L} . \\
\text { UOG- } 818\end{array}$ & Jangli jute & $\mathrm{H}$ & Wild & $\begin{array}{l}\text { Whole plant, } \\
\text { Leaves, } \\
\text { Seeds }\end{array}$ & Extract, Sap & 0.4 & 0.06 & $\begin{array}{l}\text { Gonorrhea, stomach ache, pneumonia, urethral } \\
\text { discharges, headache. }\end{array}$ \\
\hline Cuscutaceae & $\begin{array}{l}\text { Cuscuta reflexa Roxb. } \\
\text { UOG-819 }\end{array}$ & Neela tari & $\mathrm{H}$ & Wild & Whole plant & $\begin{array}{l}\text { Powder, Juice, } \\
\text { Infusion, } \\
\text { Decoction }\end{array}$ & 0.96 & 0.48 & $\begin{array}{l}\text { Astringent, purgative, anthelmintic, baldness, } \\
\text { wound, anti-lice, hair tonic, diarrhea, itches, } \\
\text { sores, gout, joint pains, skin itching, jaundice, } \\
\text { skin diseases, cough, blood purification, fever, } \\
\text { menstruation bleeding. }\end{array}$ \\
\hline Cyperaceae & $\begin{array}{l}\text { Cyperus rotundus } \mathrm{L} \text {. } \\
\text { UOG-820 }\end{array}$ & Deela & $\mathrm{Sd}$ & Wild & $\begin{array}{l}\text { Leaves, } \\
\text { Rhizome, } \\
\text { Root }\end{array}$ & Paste, Extract & 0.76 & 0.38 & $\begin{array}{l}\text { Wounds, fever, increase milk secretion, treat } \\
\text { stomach, bowel and inflammatory diseases }\end{array}$ \\
\hline Poaceae & $\begin{array}{l}\text { Cynodon dactylon Pers. } \\
\text { UOG-821 }\end{array}$ & Khabal & $\mathrm{H}$ & Wild & Whole plant & $\begin{array}{l}\text { Decoction, } \\
\text { Juice }\end{array}$ & 0.64 & 0.26 & $\begin{array}{l}\text { Fever, syphilis, dropsy, astringent, antiseptic, liver } \\
\text { problems, cough, diarrhea, dysentery, headache } \\
\text { and stomachache, wounds, nose bleeding. }\end{array}$ \\
\hline Papilionaceae & $\begin{array}{l}\text { Dalbergia sissoo DC. } \\
\text { UOG-822 }\end{array}$ & Tali & $T$ & Wild & Leaves & $\begin{array}{l}\text { Juice, } \\
\text { Decoction }\end{array}$ & 0.45 & 0.15 & $\begin{array}{l}\text { Vomiting, piles, and obesity, eye pain, abdomen } \\
\text { pain, gonorrhea, jaundice, skin diseases. }\end{array}$ \\
\hline Solanaceae & $\begin{array}{l}\text { Datura innoxia Mill. } \\
\text { UOG-823 }\end{array}$ & Datura & $\mathrm{H}$ & Wild & Leaves & $\begin{array}{l}\text { Juice, Smoke, } \\
\text { Poultice }\end{array}$ & 0.6 & 0.2 & $\begin{array}{l}\text { Snake bite, antiseptic, sedative, narcotic, asthma, } \\
\text { dandruff, antispasmodic, boils. }\end{array}$ \\
\hline Amaranthaceae & $\begin{array}{l}\text { Digera muricata (L.) Mart } \\
\text { UOG-824 }\end{array}$ & Tandla & $\mathrm{H}$ & Wild & $\begin{array}{l}\text { Leaves, } \\
\text { Flower, Seed }\end{array}$ & $\begin{array}{l}\text { Paste, } \\
\text { Decoction }\end{array}$ & 0.73 & 0.36 & $\begin{array}{l}\text { Constipation, renal disorders, digestive } \\
\text { problems, urinary disorders. }\end{array}$ \\
\hline Sapindaceae & $\begin{array}{l}\text { Dodonaea viscosa (L.) Jacq. } \\
\text { UOG-825 }\end{array}$ & Sanatha & $\mathrm{s}$ & Wild & Leaves & $\begin{array}{l}\text { Infusion, } \\
\text { Extract, Paste }\end{array}$ & 0.75 & 0.25 & $\begin{array}{l}\text { Wound, abotificient, febrifuge, cold, sore throat, } \\
\text { itching. }\end{array}$ \\
\hline
\end{tabular}


Ethnobotany Research and Applications

\begin{tabular}{|c|c|c|c|c|c|c|c|c|c|}
\hline Euphorbiaceae & $\begin{array}{l}\text { Euphorbia hirta L. } \\
\text { UOG-826 }\end{array}$ & Dudhi kalan & $\mathrm{H}$ & Wild & $\begin{array}{l}\text { Leaves, } \\
\text { Latex, Whole } \\
\text { plant }\end{array}$ & $\begin{array}{l}\text { Paste, } \\
\text { Decoction }\end{array}$ & 0.55 & 0.41 & $\begin{array}{l}\text { Cough and asthma, diarrhea, wounds, } \\
\text { inflammations and ulcers, warts, leucorrhea, skin } \\
\text { problems. }\end{array}$ \\
\hline Euphorbiaceae & $\begin{array}{l}\text { Euphorbia prostrata Aiton. } \\
\text { UOG-827 }\end{array}$ & Dudhi & $\mathrm{H}$ & Wild & Whole plant & $\begin{array}{l}\text { Powder, Juice, } \\
\text { Chewing, } \\
\text { Decoction }\end{array}$ & 0.33 & 0.16 & $\begin{array}{l}\text { Diabetes, labor pain, warts, abscesses, abortion, } \\
\text { gonorrhea, headache. }\end{array}$ \\
\hline Moraceae & $\begin{array}{l}\text { Ficus auriculata Lour. } \\
\text { UOG-828 }\end{array}$ & Tossy & $T$ & Wild & $\begin{array}{l}\text { Leaves, } \\
\text { Fruits, Roots, } \\
\text { Latex, Bark }\end{array}$ & $\begin{array}{l}\text { Paste, Juice, } \\
\text { Powder, } \\
\text { Infusion }\end{array}$ & 0.88 & 0.36 & $\begin{array}{l}\text { High blood pressure, cuts, wounds, diarrhea, } \\
\text { dysentery, cholera, mumps, vomiting, diabetes, } \\
\text { jaundice. }\end{array}$ \\
\hline Moraceae & $\begin{array}{l}\text { Ficus benghalensis } \mathrm{L} \text {. } \\
\text { UOG-829 }\end{array}$ & Bohr & $\bar{T}$ & Wild & $\begin{array}{l}\text { Leaves, Fruit, } \\
\text { Hanging } \\
\text { root, Milky } \\
\text { latex, Bark }\end{array}$ & $\begin{array}{l}\text { Powder, Milk, } \\
\text { Extract, } \\
\text { Infusion }\end{array}$ & 0.94 & 0.55 & $\begin{array}{l}\text { Flu, spermatorrhoea, premature ejaculation, } \\
\text { sexual desire, dysentery, diarrhea, diabetes, } \\
\text { tonic, cooling. }\end{array}$ \\
\hline Moraceae & $\begin{array}{l}\text { Ficus palmata Forssk. } \\
\text { UOG-830 }\end{array}$ & Phagwar & $T$ & Wild & Fruit, Leaves & $\begin{array}{l}\text { Sap, Powder, } \\
\text { Decoction }\end{array}$ & 0.9 & 0.15 & $\begin{array}{l}\text { Laxative, demulcent, lung and bladder disorders, } \\
\text { warts, diabetes, ulcers. }\end{array}$ \\
\hline Moraceae & $\begin{array}{l}\text { Ficus rasimosa } \mathrm{L} . \\
\text { UOG- } 835\end{array}$ & Rumbal & $T$ & Wild & $\begin{array}{l}\text { Leaves, } \\
\text { Fruits, Latex, } \\
\text { Root }\end{array}$ & $\begin{array}{l}\text { Chewing, } \\
\text { Decoction, } \\
\text { Paste }\end{array}$ & 0.85 & 0.28 & $\begin{array}{l}\text { Bile, diabetes and diarrhea, piles, stomachic, } \\
\text { carminative, hemoptysis, tonsils. }\end{array}$ \\
\hline Moraceae & $\begin{array}{l}\text { Ficus religiosa L. } \\
\text { UOG-836 }\end{array}$ & Peepal & $T$ & Wild & $\begin{array}{l}\text { Bark, Fruit, } \\
\text { Leaves }\end{array}$ & $\begin{array}{l}\text { Paste, Ash, } \\
\text { Powder, } \\
\text { Infusion, } \\
\text { Decoction, } \\
\text { Extract, Sap }\end{array}$ & 0.77 & 0.45 & $\begin{array}{l}\text { Inflammation, diarrhea, dysentery, asthma, } \\
\text { infections of skin and ulcers, cholera, wound, } \\
\text { diabetes, scabies, cracked heals. }\end{array}$ \\
\hline Moraceae & $\begin{array}{l}\text { Ficus semicordata Buch.- } \\
\text { Ham. ex Sm. } \\
\text { UOG-837 }\end{array}$ & Panjal & $T$ & Wild & $\begin{array}{l}\text { Fruit, leaves, } \\
\text { Latex }\end{array}$ & Juice, Paste & 0.82 & 0.48 & $\begin{array}{l}\text { Headache, fever, menstrual disorders, } \\
\text { constipation. }\end{array}$ \\
\hline Flacourtiaceae & $\begin{array}{l}\text { Flacourtia indica (Burm. f.) } \\
\text { Merr. } \\
\text { UOG-838 }\end{array}$ & Koko & $S$ & Wild & $\begin{array}{l}\text { Fruits, } \\
\text { Leaves, } \\
\text { Roots, Bark }\end{array}$ & $\begin{array}{l}\text { Decoction, } \\
\text { Ash, Paste }\end{array}$ & 0.65 & 0.21 & $\begin{array}{l}\text { Diuretic, astringent, anthelmintic, snake bites, } \\
\text { body pain, kidney problems, cough, diarrhea, } \\
\text { arthritis, digestion, appetizer, jaundice, vomiting, } \\
\text { nausea, headache, }\end{array}$ \\
\hline Rubiaceae & $\begin{array}{l}\text { Galium aparine L. } \\
\text { UOG-839 }\end{array}$ & Kori buti & $\mathrm{H}$ & Wild & $\begin{array}{l}\text { Leaves,Whol } \\
\text { e plant }\end{array}$ & $\begin{array}{l}\text { Paste, Juice, } \\
\text { Extract }\end{array}$ & 0.26 & 0.06 & $\begin{array}{l}\text { Kidney stones, skin troubles, face wash, wound, } \\
\text { stop bleeding, anticancer. }\end{array}$ \\
\hline Colchiaceae & $\begin{array}{l}\text { Gloriosa superba L. } \\
\text { UOG-840 }\end{array}$ & Charkiara & $\mathrm{H}$ & Wild & $\begin{array}{l}\text { Tuber, Root, } \\
\text { Leaves }\end{array}$ & $\begin{array}{l}\text { Paste, Juice, } \\
\text { Ash, } \\
\text { Decoction, Sap }\end{array}$ & 0.6 & 0.03 & $\begin{array}{l}\text { Stimulant, abortifacient, anthelmintic, leprosy, } \\
\text { antidote to snake poison, earache, toothache, } \\
\text { asthma, wounds, pimples, malaria, abdominal } \\
\text { problems, reduce abortion. }\end{array}$ \\
\hline Tiliaceae & $\begin{array}{l}\text { Grewia optiva J. R. Drumm. } \\
\text { UOG-841 }\end{array}$ & Dahman & $T$ & Wild & $\begin{array}{l}\text { Leaves, Fruit, } \\
\text { Bark }\end{array}$ & Extract, Sap & 0.46 & 0.23 & $\begin{array}{l}\text { Smooth delivery, constipation, oxidative stress, } \\
\text { neurological disorders, diabetes. }\end{array}$ \\
\hline Celasteraceae & $\begin{array}{l}\text { Gymnosporea royleana Wall. } \\
\text { ex M. A. Lawson } \\
\text { UOG-842 }\end{array}$ & Pataki & $S$ & Wild & $\begin{array}{l}\text { Leaves, } \\
\text { Seeds, Roots }\end{array}$ & $\begin{array}{l}\text { Powder, } \\
\text { Decoction, } \\
\text { Infusion, Sap }\end{array}$ & 0.33 & 0.16 & $\begin{array}{l}\text { Gastro-intestinal problems, dysentery, } \\
\text { vermifuge, toothache, catarrh, sores, mouth } \\
\text { infections, eye problems. }\end{array}$ \\
\hline
\end{tabular}


Ethnobotany Research and Applications

\begin{tabular}{|c|c|c|c|c|c|c|c|c|c|}
\hline Araliaceae & $\begin{array}{l}\text { Hedera nepalensis K. Koch } \\
\text { UOG-843 }\end{array}$ & Banjali & $\begin{array}{l}\text { Climbi } \\
\text { ng } \\
\text { shrub }\end{array}$ & Wild & Leaves, Fruit & Decoction & 0.53 & 0.26 & $\begin{array}{l}\text { Cathartic, stimulant, diaphoretic, febrile } \\
\text { disorders, rheumatism, skin problems, diabetes. }\end{array}$ \\
\hline Convolvulaceae & $\begin{array}{l}\text { Ipomea pes-tigridis L. } \\
\text { UOG-844 }\end{array}$ & Pulichuvali & $\mathrm{H}$ & Wild & $\begin{array}{l}\text { Leaves, } \\
\text { Seeds, } \\
\text { Roots, } \\
\text { Whole plant }\end{array}$ & $\begin{array}{l}\text { Poultice, } \\
\text { Smoke, Juice, } \\
\text { Decoction }\end{array}$ & 0.4 & 0.06 & $\begin{array}{l}\text { Boils, sores, pimples and tumors, bronchial } \\
\text { spasm, dropsy, rabies dog bites, hemoptysis. }\end{array}$ \\
\hline Acanthaceae & $\begin{array}{l}\text { Justicia adhatoda L. } \\
\text { UOG-845 }\end{array}$ & Baikar & $S$ & Wild & Leaves, Root & $\begin{array}{l}\text { Juice, } \\
\text { Decoction }\end{array}$ & 0.8 & 0.33 & $\begin{array}{l}\text { Inflammation, antispasmodic, expectorant, } \\
\text { cough, pneumonia, dysentery, diarrhea, } \\
\text { glandular tumors. }\end{array}$ \\
\hline Plantaginaceae & $\begin{array}{l}\text { Nanorrhinum ramossisimum } \\
\text { (Wall.) Betsche. } \\
\text { UOG-846 }\end{array}$ & $\begin{array}{l}\text { Khunger } \\
\text { booti }\end{array}$ & $\mathrm{H}$ & Wild & Whole plant & Powder, Paste & 0.56 & 0.28 & $\begin{array}{l}\text { Diabetes, diuretic, break kidney stones, fever, } \\
\text { rheumatism, snake and scorpion bites, } \\
\text { inflammation. }\end{array}$ \\
\hline Anacardiaceae & $\begin{array}{l}\text { Lannea coromandelica } \\
\text { (Houtt.) Merr. } \\
\text { UOG-847 }\end{array}$ & Kamliya & $T$ & Wild & $\begin{array}{l}\text { Resin, Bark, } \\
\text { Fruit, Leaves }\end{array}$ & $\begin{array}{l}\text { Powder, Juice, } \\
\text { Paste }\end{array}$ & 0.46 & 0.23 & $\begin{array}{l}\text { Dysentery, fever, stomach pain, astringent, } \\
\text { toothache, gum problems, inflammation, body } \\
\text { pains, wounds, skin diseases, stop bleeding, } \\
\text { tetanus. }\end{array}$ \\
\hline Mimosaceae & $\begin{array}{l}\text { Leucaena leucocephala } \\
\text { (Lam.) de Wit. } \\
\text { UOG-848 }\end{array}$ & Babul & $T$ & Wild & $\begin{array}{l}\text { Leaves, } \\
\text { Root, Bark, } \\
\text { Seeds }\end{array}$ & $\begin{array}{l}\text { Decoction, } \\
\text { Paste }\end{array}$ & 0.66 & 0.16 & $\begin{array}{l}\text { Cough, measles, intestinal worms, abortifacient, } \\
\text { diabetes. }\end{array}$ \\
\hline Brassicaceae & $\begin{array}{l}\text { Lepedium sativum } \mathrm{L} . \\
\text { UOG-849 }\end{array}$ & Halon & $\mathrm{H}$ & Cultivated & $\begin{array}{l}\text { Leaves, } \\
\text { Seeds }\end{array}$ & $\begin{array}{l}\text { Chewed, Paste, } \\
\text { Seeds, } \\
\text { Decoction }\end{array}$ & 0.71 & 0.41 & $\begin{array}{l}\text { Cough, asthma, sore throat and headache, } \\
\text { chapped lips, sunburn, liver disorders, appetizer, } \\
\text { digestive disorders. }\end{array}$ \\
\hline Euphorbiaceae & $\begin{array}{l}\text { Mallotus philippensis (Lam.) } \\
\text { Mull. Arg. } \\
\text { UOG-850 }\end{array}$ & Kamela & $T$ & Wild & $\begin{array}{l}\text { Leaves,Fruit, } \\
\text { Root, Bark }\end{array}$ & $\begin{array}{l}\text { Powder, Juice, } \\
\text { Decoction, } \\
\text { Paste }\end{array}$ & 0.88 & 0.51 & $\begin{array}{l}\text { Measles, mumps, threadworms, ascaris, } \\
\text { anthelmintic, cathartic, purgative, carminative, } \\
\text { bronchitis, jaundice, spleen enlargement, piles, } \\
\text { skin diseases, ulcer, diarrhea. }\end{array}$ \\
\hline Malvaceae & $\begin{array}{l}\text { Malva parviflora L. } \\
\text { UOG-851 }\end{array}$ & Sonchal & $\mathrm{H}$ & Wild & $\begin{array}{l}\text { Whole plant, } \\
\text { Seeds, Roots }\end{array}$ & $\begin{array}{l}\text { Poultice, } \\
\text { Decoction, } \\
\text { Seeds }\end{array}$ & 0.68 & 0.28 & $\begin{array}{l}\text { Inflammations, sores, boils, cough, bladder ulcer, } \\
\text { dandruff, demulcent. }\end{array}$ \\
\hline Malvaceae & $\begin{array}{l}\text { Malvastrum } \\
\text { coromandelianum (L.) } \\
\text { Garcke } \\
\text { UOG-852 }\end{array}$ & Baddi Brear & $\mathrm{S}$ & Wild & $\begin{array}{l}\text { Leaves, } \\
\text { Flowers, } \\
\text { Rhizome }\end{array}$ & $\begin{array}{l}\text { Paste, } \\
\text { Decoction, } \\
\text { Powder }\end{array}$ & 0.4 & 0.06 & $\begin{array}{l}\text { Dysentery, sores, inflammations, wounds, } \\
\text { diaphoretic, carbuncles, dysentery, cough, lungs } \\
\text { diseases, jaundice, fever, diarrhea, muscular pain. }\end{array}$ \\
\hline Martyniaceae & $\begin{array}{l}\text { Martynia annua L. } \\
\text { UOG-853 }\end{array}$ & Bhindoo & $\mathrm{S}$ & Wild & Leaves, Fruit & Juice, Paste & 0.25 & 0.083 & $\begin{array}{l}\text { Epilepsy, sore throat, alexetric, insects bites, } \\
\text { wounds. }\end{array}$ \\
\hline Meliaceae & $\begin{array}{l}\text { Melia azedarach L. } \\
\text { UOG-854 }\end{array}$ & Dahrek & $T$ & Wild & $\begin{array}{l}\text { Leaves, fruit, } \\
\text { Bark, Root, } \\
\text { Heart wood }\end{array}$ & $\begin{array}{l}\text { Juice, Pulp, } \\
\text { Infusion, } \\
\text { Extract, Paste }\end{array}$ & 0.95 & 0.31 & $\begin{array}{l}\text { Blood purification, tonic for stomach and liver, } \\
\text { spleen enlargement, ascariasis, dandruff, eczema, } \\
\text { ringworms, skin itching, malaria, antiseptic and } \\
\text { febrifuge. }\end{array}$ \\
\hline
\end{tabular}


Ethnobotany Research and Applications

\begin{tabular}{|c|c|c|c|c|c|c|c|c|c|}
\hline Lamiaceae & $\begin{array}{l}\text { Mentha longifolia (L.) L. } \\
\text { UOG-855 }\end{array}$ & $\begin{array}{l}\text { Jangli } \\
\text { Podina }\end{array}$ & $\mathrm{H}$ & Wild & $\begin{array}{l}\text { Leaves, } \\
\text { Flowering } \\
\text { stem }\end{array}$ & $\begin{array}{l}\text { Powder, } \\
\text { Extract, Paste, } \\
\text { Tea }\end{array}$ & 0.92 & 0.38 & $\begin{array}{l}\text { Flu, fever, vomiting, dysentery, asthma, } \\
\text { antiseptic, refrigerant, carminative, stimulant, } \\
\text { diuretic, liver and spleen disorders, wounds. }\end{array}$ \\
\hline Moringaceae & $\begin{array}{l}\text { Moringa oleifera Lam. } \\
\text { UOG-856 }\end{array}$ & Suhanjana & $T$ & Cultivated & $\begin{array}{l}\text { Leaves, } \\
\text { Pods, } \\
\text { Flowers, } \\
\text { Roots }\end{array}$ & $\begin{array}{l}\text { Pills, Extract, } \\
\text { Pickle }\end{array}$ & 0.85 & 0.28 & $\begin{array}{l}\text { Diabetes, rubefacient and vasccant, antipyretic, } \\
\text { stomach disorders, high blood pressure. }\end{array}$ \\
\hline Moraceae & $\begin{array}{l}\text { Morus nigra L. } \\
\text { UOG- } 857\end{array}$ & Kala toot & $T$ & Wild & $\begin{array}{l}\text { Leaves, Fruit, } \\
\text { Bark, Root }\end{array}$ & $\begin{array}{l}\text { Decoction, } \\
\text { Juice, Raw fruit }\end{array}$ & 0.9 & 0.15 & $\begin{array}{l}\text { Cough, anthelmintic, purgative, vermifuge, } \\
\text { diarrhea, anemia, digestion, strengthen bones, } \\
\text { improve immunity, cardiovascular disorders. }\end{array}$ \\
\hline Apocynaceae & $\begin{array}{l}\text { Nerium oleander } \mathrm{L} . \\
\text { UOG-858 }\end{array}$ & Gandira & $\mathrm{S}$ & Wild & $\begin{array}{l}\text { Bark, Leaves, } \\
\text { Roots }\end{array}$ & $\begin{array}{l}\text { Paste, Oil, } \\
\text { Decoction, } \\
\text { Powder }\end{array}$ & 0.36 & 0.18 & $\begin{array}{l}\text { Arithritis, heart tonic, hemorrhoids, ulcers, skin } \\
\text { diseases, scabies, inflammation, epilepsy, } \\
\text { leprosy. }\end{array}$ \\
\hline Oleaceae & $\begin{array}{l}\text { Olea ferruginea Wall. ex } \\
\text { Aitch. } \\
\text { UOG-859 }\end{array}$ & Kao & $T$ & Wild & Leaves, roots & $\begin{array}{l}\text { Extract, } \\
\text { Decoction, Tea }\end{array}$ & 0.84 & 0.35 & $\begin{array}{l}\text { Diabetes, toothache, antiseptic, astringent, } \\
\text { stomachache, fever, malaria. }\end{array}$ \\
\hline Lamiaceae & $\begin{array}{l}\text { Rydingia limbata (Benth.) } \\
\text { Scheen \& V.A.Albert } \\
\text { UOG-860 }\end{array}$ & Chiti Ptaki & $\mathrm{S}$ & Wild & Leaves & $\begin{array}{l}\text { Powder, } \\
\text { Extract }\end{array}$ & 0.33 & 0.16 & $\begin{array}{l}\text { Mouth ulcer, wounds, mouth sores, throat pains, } \\
\text { eye and skin diseases. }\end{array}$ \\
\hline Oxalidaceae & $\begin{array}{l}\text { Oxalis corniculata } \mathrm{L} \text {. } \\
\text { UOG-861 }\end{array}$ & Khati buti & $\mathrm{H}$ & Wild & $\begin{array}{l}\text { Leaves, } \\
\text { Shoot, } \\
\text { Whole plant }\end{array}$ & $\begin{array}{l}\text { Paste, Extract, } \\
\text { Juice }\end{array}$ & 0.75 & 0.25 & $\begin{array}{l}\text { Scurvy, appetite, malaria, jaundice, hepatitis B, } \\
\text { blood purification, abdominal pain, boils, } \\
\text { abscesses wound, weeping eczema, } \\
\text { inflammation. }\end{array}$ \\
\hline Asclepiadaceae & $\begin{array}{l}\text { Periploca aphylla Decne. } \\
\text { UOG-862 }\end{array}$ & Batta & $\mathrm{S}$ & Wild & $\begin{array}{l}\text { Bark, Milky } \\
\text { juice }\end{array}$ & $\begin{array}{l}\text { Decoction, } \\
\text { Juice, Paste }\end{array}$ & 0.46 & 0.11 & $\begin{array}{l}\text { Purgative, stomachic, fever, blisters of pus and } \\
\text { tumors stomachic, fever. }\end{array}$ \\
\hline Euphorbiaceae & $\begin{array}{l}\text { Phyllanthes emblica L. } \\
\text { UOG-863 }\end{array}$ & Amla & $T$ & Wild & Fruit & Powder, Juice & 0.93 & 0.23 & $\begin{array}{l}\text { Diabetes, high blood pressure, cooling, } \\
\text { astringent, laxative, diuretic, diarrhea, dysentery } \\
\text { and eye problems. }\end{array}$ \\
\hline Euphorbiaceae & $\begin{array}{l}\text { Phyllanthus niruri L. } \\
\text { UOG-864 }\end{array}$ & Jangli amli & $\mathrm{H}$ & Wild & $\begin{array}{l}\text { Fruit, Leaves, } \\
\text { Root, Seeds }\end{array}$ & $\begin{array}{l}\text { Poultice, } \\
\text { Infusion, Paste, } \\
\text { Seeds }\end{array}$ & 0.35 & 0.11 & $\begin{array}{l}\text { Stones of urinary tract, dysentery, ulcers, } \\
\text { inflammations, diuretic, diabetes, sores, wounds, } \\
\text { ringworms, febrifuge, bruises. }\end{array}$ \\
\hline Solanaceae & $\begin{array}{l}\text { Physalis minima L. } \\
\text { UOG-865 }\end{array}$ & Sun berry & $\mathrm{H}$ & Wild & $\begin{array}{l}\text { Fruit, Leaves, } \\
\text { Root, Whole } \\
\text { plant }\end{array}$ & $\begin{array}{l}\text { Juice, Extract, } \\
\text { Paste, Chewed, } \\
\text { Decoction }\end{array}$ & 0.48 & 0.2 & $\begin{array}{l}\text { Appetizer, diuretic, laxative, tonic, anticancer, } \\
\text { earache, headache, itches, febrifuge, vermifuge, } \\
\text { abdomen pain. }\end{array}$ \\
\hline Pinaceae & $\begin{array}{l}\text { Pinus roxburghii Sarg. } \\
\text { UOG-866 }\end{array}$ & Cheer & $T$ & Wild & $\begin{array}{l}\text { Leaves, Fruit, } \\
\text { Resin }\end{array}$ & Extract & 0.6 & 0.3 & $\mathrm{~TB}$, chronic bronchitis and gangrin of lungs. \\
\hline Plumbaginaceae & $\begin{array}{l}\text { Plumbago zeylanica L. } \\
\text { UOG-567 }\end{array}$ & Chitra & $\mathrm{S}$ & Wild & $\begin{array}{l}\text { Whole plant, } \\
\text { Roots, } \\
\text { Leaves }\end{array}$ & $\begin{array}{l}\text { Juice, } \\
\text { Decoction, } \\
\text { Powder, Paste }\end{array}$ & 0.25 & 0.083 & $\begin{array}{l}\text { Tuberculosis, syphilis, gonorrhea, throat, mouth } \\
\text { and chest inflammations, diarrhea, dyspepsia, } \\
\text { piles, scabies. }\end{array}$ \\
\hline
\end{tabular}


Ethnobotany Research and Applications

\begin{tabular}{|c|c|c|c|c|c|c|c|c|c|}
\hline Fabaceae & $\begin{array}{l}\text { Pueraria tuberosa (Willd.) } \\
\text { DC. } \\
\text { UOG-868 }\end{array}$ & Bandhari & $\begin{array}{l}\text { Climbi } \\
\text { ng } \\
\text { Shrub }\end{array}$ & Wild & Root, Tuber & Paste, Powder & 0.8 & 0.33 & $\begin{array}{l}\text { Cuts, inflammation, epilepsy, sperm count, skin } \\
\text { complexion. }\end{array}$ \\
\hline Euphorbiaceae & $\begin{array}{l}\text { Ricinus communis } \mathrm{L} . \\
\text { UOG-869 }\end{array}$ & Arnoli & $\mathrm{S}$ & Wild & Seeds & Paste, oil & 0.86 & 0.21 & $\begin{array}{l}\text { Constipation, leprosy, syphilis, inflammation, } \\
\text { carbuncles and boils, control birth. }\end{array}$ \\
\hline Rhamnaceae & $\begin{array}{l}\text { Rhamnus triquetra (Wall) } \\
\text { Brandis } \\
\text { UOG-870 }\end{array}$ & Gount & $\mathrm{S}$ & Wild & Bark, Fruit & Juice, Paste & 0.2 & 0.05 & Diarrhea, dysentery, purify blood, scabies, boils. \\
\hline Rosaceae & $\begin{array}{l}\text { Rubus ellipticus Sm. } \\
\text { UOG-871 }\end{array}$ & Akhre & $\mathrm{S}$ & Wild & Fruit, Root & Paste, Juice & 0.45 & 0.26 & $\begin{array}{l}\text { Carminative, tonic, wounds, fever, gastric } \\
\text { problems, diarrhea, dysentery, cough, colic and } \\
\text { sore throat, astringent. }\end{array}$ \\
\hline Poaceae & $\begin{array}{l}\text { Saccharum benghalense } \\
\text { Retz. } \\
\text { UOG-872 }\end{array}$ & Saroot & $\mathrm{H}$ & Wild & $\begin{array}{l}\text { Aerial part, } \\
\text { Root }\end{array}$ & Extract & 0.6 & 0.3 & $\begin{array}{l}\text { Astringent, refrigerant, emollient, diuretic, } \\
\text { purgative, aphrodisiac, treat burning sensation, } \\
\text { dyspepsia, sexual weakness, piles, respiratory } \\
\text { problems and gynaecological. }\end{array}$ \\
\hline Asteraceae & $\begin{array}{l}\text { Saussurea heteromalla (D. } \\
\text { Don) Handel-Mazzetti } \\
\text { UOG-873 }\end{array}$ & Kali ziri & $\mathrm{H}$ & Wild & $\begin{array}{l}\text { Leaves, } \\
\text { Roots, Seeds }\end{array}$ & Paste, Extract & 0.4 & 0.2 & $\begin{array}{l}\text { Leucoderma, wounds, fever, colic, carminative, } \\
\text { horse-bites. }\end{array}$ \\
\hline Caryophyllaceae & $\begin{array}{l}\text { Silene conoidea } \mathrm{L} . \\
\text { UOG- } 874\end{array}$ & Dabri & $\mathrm{H}$ & Wild & $\begin{array}{l}\text { Leaves, } \\
\text { whole plant }\end{array}$ & Juice & 0.33 & 0.16 & Ophthalmia, wounds, emollient, fumigant. \\
\hline Asteraceae & $\begin{array}{l}\text { Silybum marianum (L.) } \\
\text { Gaertn. } \\
\text { UOG-875 }\end{array}$ & Kandyari & $\mathrm{H}$ & Wild & $\begin{array}{l}\text { Leaves, } \\
\text { Seeds }\end{array}$ & $\begin{array}{l}\text { Infusion, } \\
\text { Extract, Seeds }\end{array}$ & 0.73 & 0.18 & $\begin{array}{l}\text { Chest, throat infections, stimulant, expectorant, } \\
\text { liver and gallbladder diseases, liver disorders, } \\
\text { skin protection. }\end{array}$ \\
\hline Brassicaceae & $\begin{array}{l}\text { Sisybrium irio L. } \\
\text { UOG-876 }\end{array}$ & Khoob kalan & $\mathrm{H}$ & Wild & $\begin{array}{l}\text { Seeds, Aerial } \\
\text { parts }\end{array}$ & $\begin{array}{l}\text { Decoction, } \\
\text { Poultice, } \\
\text { Infusion, } \\
\text { Extract, Seeds }\end{array}$ & 0.45 & 0.26 & $\begin{array}{l}\text { Measles, small pox, typhoid fever, throat and } \\
\text { chest affections, cholera, diarrhea, inflammation } \\
\text { and wounds. }\end{array}$ \\
\hline Solanaceae & $\begin{array}{l}\text { Solanum americanum Mill. } \\
\text { UOG-887 }\end{array}$ & Kachmach & $\mathrm{H}$ & Wild & $\begin{array}{l}\text { Leaves, Fruit, } \\
\text { Stems, Roots }\end{array}$ & $\begin{array}{l}\text { Decoction, } \\
\text { Juice, Extract, } \\
\text { Infusion, } \\
\text { Tincture }\end{array}$ & 0.83 & 0.41 & $\begin{array}{l}\text { Digestive problems, jaundice, ulcer, skin } \\
\text { problems dysentery, asthma, fever, boils, sores, } \\
\text { wounds and leucoderma, toothache, neurologic } \\
\text { pains, eye diseases, diarrhea, rabies, headache } \\
\text { and ringworm. }\end{array}$ \\
\hline Solanaceae & $\begin{array}{l}\text { Solanum surattense Burm. } f . \\
\text { UOG- } 888\end{array}$ & Mokri & $\mathrm{H}$ & Wild & $\begin{array}{l}\text { Fruit, Leaves, } \\
\text { Root }\end{array}$ & $\begin{array}{l}\text { Decoction, } \\
\text { Paste }\end{array}$ & 0.86 & 0.43 & $\begin{array}{l}\text { Pneumonia, joints pain and other body pain, } \\
\text { scabies, ringworm, expectorant, skin diseases. }\end{array}$ \\
\hline Myrtaceae & $\begin{array}{l}\text { Syzygium cumini (L.) Skeels. } \\
\text { UOG-889 }\end{array}$ & Tala & $T$ & $\begin{array}{l}\text { Wild/Cultiva } \\
\text { ted }\end{array}$ & $\begin{array}{l}\text { Fruit, Seeds, } \\
\text { Bark }\end{array}$ & $\begin{array}{l}\text { Powder, Juice, } \\
\text { Extract }\end{array}$ & 0.9 & 0.45 & $\begin{array}{l}\text { Diabetes, diarrhea, asthma, bronchitis, ulcers, } \\
\text { diarrhea and dysentery. }\end{array}$ \\
\hline Combretaceae & $\begin{array}{l}\text { Terminalia bellirica (Gaertn) } \\
\text { Roxb. } \\
\text { U0G-890 }\end{array}$ & Baira & $T$ & Wild & $\begin{array}{l}\text { Fruit, Bark, } \\
\text { Gum, Seed }\end{array}$ & $\begin{array}{l}\text { Paste, Fruit, } \\
\text { powder }\end{array}$ & 0.84 & 0.35 & $\begin{array}{l}\text { Asthma, astringent, purgative, rheumatic } \\
\text { swellings, diuretic, demulcent, inflammation, } \\
\text { digestive, respiratory disorders, constipation. }\end{array}$ \\
\hline
\end{tabular}


Ethnobotany Research and Applications

\begin{tabular}{|c|c|c|c|c|c|c|c|c|c|}
\hline Menispermaceae & $\begin{array}{l}\text { Tinospora sinensis (Lour.) } \\
\text { Merr. } \\
891\end{array}$ & Garon & $\begin{array}{l}\text { Climbi } \\
\text { ng } \\
\text { Shrub }\end{array}$ & Wild & $\begin{array}{l}\text { Stem, Bark, } \\
\text { Root }\end{array}$ & $\begin{array}{l}\text { Extract, Juice, } \\
\text { Chew, Powder }\end{array}$ & 0.93 & 0.23 & $\begin{array}{l}\text { Fever, aphrodisiac, antiperiodic, diarrhea, } \\
\text { arithritis, asthma, diabetes. }\end{array}$ \\
\hline Asteraceae & $\begin{array}{l}\text { Traxacum campylodesG. E. } \\
\text { Haglund } \\
\text { UOG-892 }\end{array}$ & Peeli buti & $\mathrm{H}$ & Wild & $\begin{array}{l}\text { Leaves, } \\
\text { Roots, Fruits, } \\
\text { Latex }\end{array}$ & $\begin{array}{l}\text { Paste, Juice, } \\
\text { Decoction, } \\
\text { Extract }\end{array}$ & 0.36 & 0.18 & $\begin{array}{l}\text { Inflammation, joints pain, diabetes, heart } \\
\text { problems, liver disorders, intermittent fever, } \\
\text { chronic hepatitis, hypochondria, laxative, } \\
\text { promote appetite, jaundice and purify blood, } \\
\text { boils and other skin infections. }\end{array}$ \\
\hline Asteraceae & $\begin{array}{l}\text { Tridax procumbens }(\mathrm{L} .) \mathrm{L} . \\
\text { UOG-893 }\end{array}$ & Kanphuli & $\mathrm{H}$ & Wild & Leaves & $\begin{array}{l}\text { Powder, Paste, } \\
\text { Sap, Decoction }\end{array}$ & 0.25 & 0.083 & $\begin{array}{l}\text { Antiseptic, dysentery, bronchial catarrh, diarrhea, } \\
\text { diabetes, inflammation, bleeding, sores and } \\
\text { ulcers. }\end{array}$ \\
\hline Boraginaceae & $\begin{array}{l}\text { Trichodesma indicum (L.) } \\
\text { Lehm. } \\
\text { UOG-894 }\end{array}$ & Handusi & $\mathrm{H}$ & Wild & $\begin{array}{l}\text { Roots, } \\
\text { leaves, } \\
\text { whole plant }\end{array}$ & Juice, Paste & 0.5 & 0.25 & $\begin{array}{l}\text { Ear pain, wound, cough, cold, fever, vomiting } \\
\text { and urinary problems, dysentery, wounds, joints } \\
\text { pain, inflammation. }\end{array}$ \\
\hline Scrophulariaceae & $\begin{array}{l}\text { Verbascum thapsus } \mathrm{L} . \\
\text { UOG-895 }\end{array}$ & $\begin{array}{l}\text { Gidar } \\
\text { tobacco }\end{array}$ & $\mathrm{H}$ & Wild & $\begin{array}{l}\text { Leaves, } \\
\text { Inflorescenc } \\
\text { e, Root }\end{array}$ & $\begin{array}{l}\text { Paste, Smoke, } \\
\text { Tea, Ash, } \\
\text { Decoction }\end{array}$ & 0.73 & 0.36 & $\begin{array}{l}\text { Asthma, sore throat, cold, dysentery, } \\
\text { hemorrhoids, inflammation, sunburn, cough, } \\
\text { asthma, wounds. }\end{array}$ \\
\hline Violaceae & $\begin{array}{l}\text { Viola canescens Wall. } \\
\text { UOG-896 }\end{array}$ & Banfsha & $\mathrm{H}$ & Wild & $\begin{array}{l}\text { Flower, Root, } \\
\text { Whole plant }\end{array}$ & $\begin{array}{l}\text { Powder, } \\
\text { Decoction, } \\
\text { Extract }\end{array}$ & 0.91 & 0.53 & $\begin{array}{l}\text { Fever, flu, constipation, laxative, skin abrations, } \\
\text { jaundice. }\end{array}$ \\
\hline Lythraceae & $\begin{array}{l}\text { Woodfordia fruticosa (L.) } \\
\text { Kurz. } \\
\text { UOG-897 }\end{array}$ & Tavi & $\mathrm{S}$ & Wild & $\begin{array}{l}\text { Flowers, } \\
\text { Leaves, } \\
\text { Roots }\end{array}$ & $\begin{array}{l}\text { Smoke, } \\
\text { Extract, } \\
\text { Powder, Juice }\end{array}$ & 0.66 & 0.16 & $\begin{array}{l}\text { Vermifuge catarrh and headache, fever, } \\
\text { tranquilizer, anticancer, dysentery, skin burning, } \\
\text { headache. }\end{array}$ \\
\hline Solanaceae & $\begin{array}{l}\text { Withania somnifera (L.) } \\
\text { Dunal. } \\
\text { UOG-898 }\end{array}$ & Ashgand & $\mathrm{s}$ & Wild & $\begin{array}{l}\text { Root, Fruit, } \\
\text { Leaves }\end{array}$ & $\begin{array}{l}\text { Powder, } \\
\text { Extract, Paste }\end{array}$ & 0.93 & 0.46 & $\begin{array}{l}\text { Diuretic, tonic and aphrodisiac, sexual problems } \\
\text { and promotes urination, spermatorrhoea, } \\
\text { narcotics, ulcer, swelling, diuretic, joints pains, } \\
\text { skin sore, inflammations, insomnia, stress and } \\
\text { anxiety. }\end{array}$ \\
\hline Rubiaceae & $\begin{array}{l}\text { Wendlandia heynei (Schult.) } \\
\text { Santapau \& Merchant } \\
\text { UOG-899 }\end{array}$ & Okan & $T$ & Wild & $\begin{array}{l}\text { Leaves, } \\
\text { Fruits }\end{array}$ & $\begin{array}{l}\text { Powder, } \\
\text { Decoction }\end{array}$ & 0.43 & 0.21 & $\begin{array}{l}\text { Heal wounds, amenorrhea, antispasmodic and } \\
\text { febrifuge. }\end{array}$ \\
\hline Rutaceae & $\begin{array}{l}\text { Zanthoxylum armatum DC. } \\
\text { UOG-900 }\end{array}$ & Timber & $\mathrm{S}$ & Wild & Leaves,Fruit & $\begin{array}{l}\text { Chewing, } \\
\text { Paste }\end{array}$ & 0.9 & 0.45 & $\begin{array}{l}\text { Stomach problems, condiment and carminative, } \\
\text { toothache, tonic, stimulant and antirheumatic, } \\
\text { musche spasms, chest problems, leucoderma. }\end{array}$ \\
\hline Rhamnaceae & $\begin{array}{l}\text { Ziziphus jujuba Mill. } \\
\text { UOG-901 }\end{array}$ & Jahnd & $T$ & Wild & $\begin{array}{l}\text { Leaves, Fruit, } \\
\text { Root }\end{array}$ & $\begin{array}{l}\text { Decoction, } \\
\text { Powder }\end{array}$ & 0.45 & 0.26 & $\begin{array}{l}\text { Diabetes, menorrhagia, haemorrhages, seminal } \\
\text { weakness, dyspepsia, fever, wounds, ulcers. }\end{array}$ \\
\hline Rhamnaceae & $\begin{array}{l}\text { Ziziphus oxyphylla Edge. } \\
\text { UOG-902 }\end{array}$ & Mimyaon & $\mathrm{S}$ & Wild & $\begin{array}{l}\text { Root, Fruit, } \\
\text { Leaves }\end{array}$ & $\begin{array}{l}\text { Decoction, } \\
\text { Juice }\end{array}$ & 0.84 & 0.35 & $\begin{array}{l}\text { Jaundice, diabetes and liver problems, } \\
\text { hypertension and gas troubles, allergy, intestinal } \\
\text { worms. }\end{array}$ \\
\hline
\end{tabular}




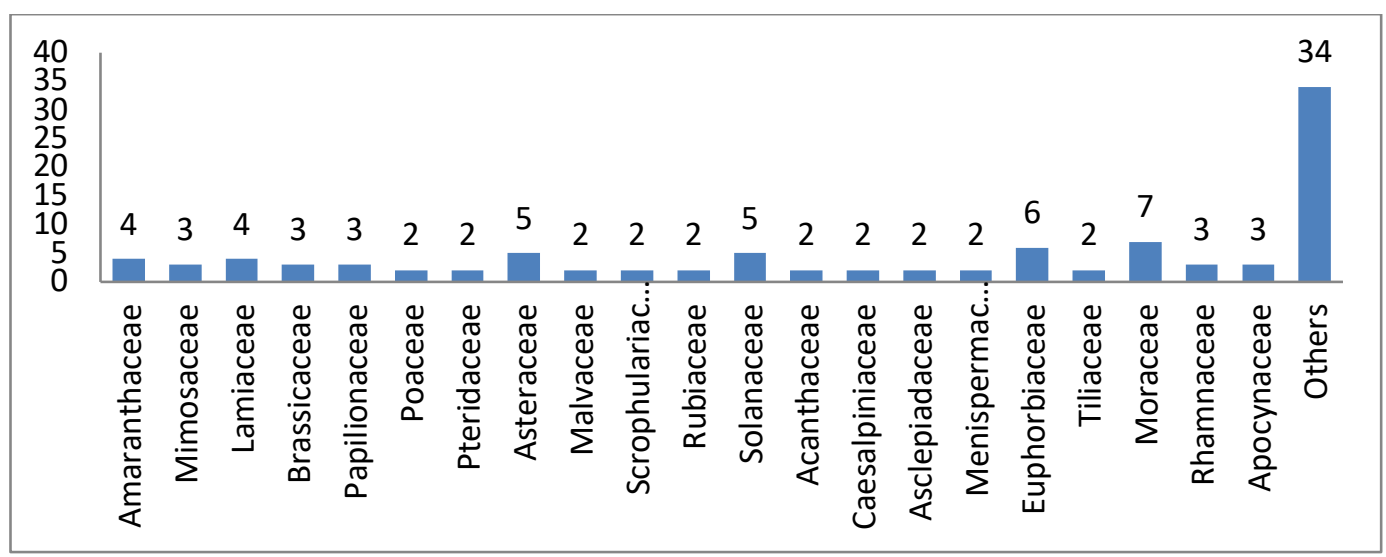

Figure 2. Graphical representation of family index (FI) with highest number of species used in present study area

\section{Diversity of plant parts used}

Total 16 plant parts were used in present study to prepare various traditional remedies for the cure of diverse ailments. The most frequently used plant parts for preparation of herbal remedies were leaves (80 species, $28 \%$ ), followed by roots (53 species, 18\%), fruits (34 species, 12\%), bark and seeds ( 23 species, $8 \%$ in each), whole plant (22 species, 8\%), stem (13 species, 4\%), flower (10 species, 3\%), wood and latex (9 species, 3\% in each), resin (3 species, 1\%), rhizome, pods, tuber, gum and aerial parts (2 species, 1\% in each) (Figure 3). In most of the cases more than one part of the same plants were used for preparation of different herbal remedies. Similar findings were also recorded by the earlier studies which had been conducted in the region of Azad Jammu and Kashmir and Indonesia (Ishtiaq et al. 2021, Supiandi et al. 2019). Leaves are widely utilized in preparation of herbal medicines due to presence of active secondary metabolites (Ghorbani 2005, Bano et al. 2014) and leaves also useful for preparation of various herbal products in many communities around the world. Similarly leaves were dominant plant part used for preparation of herbal medicines in Kel village, Azad Jammu and Kashmir (Ahmad et al. 2017). The leaves are the most common and preferred components used in medical preparations due to their easy handling and stability (Rodrigues et al. 2020).

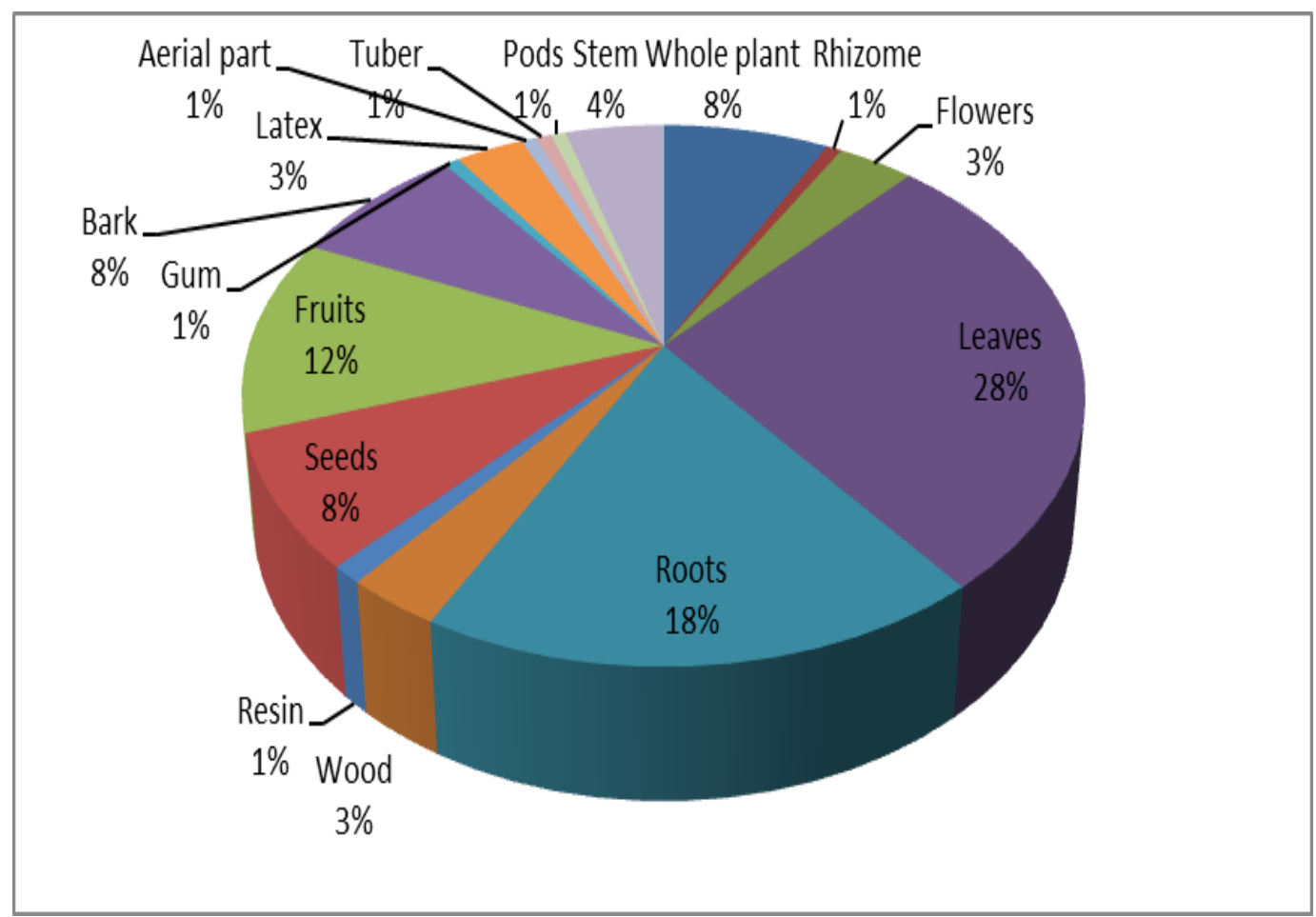

Figure 3. Percentage of plant parts used for preparation of herbal remedies 


\section{Diversity in mode of administration of herbal remedies}

In present study total 18 kinds of mode of preparation of herbal remedies were used to cure various diseases. The most frequently preparation methods used for herbal remedies were paste (59 records, 59\%), followed by decoction (54 records, 54\%), powder and extract (34 records, 34\% in each), juice (49 records, 49\%), infusion (15 records, 15\%), poultice (11 records, 11\%), sap (8 records, 8\%), Chewing (7 records, 7\%), ash (5 records, 5\%), oil (3 records, 3\%), pickles (2 records, $2 \%)$, pills and tincture (1 records, $1 \%$ in each) (Figure 5$)$. The dosage of herbal remedies were varied on the basis of disease type, age of patients, physical health of patients, severity of disease, and experience of herbal practitioners (hakims). Some of the plant parts were used with other ingredients like honey, water, butter or milk to cure various diseases. Faruque et al. (2018) reported that paste was the first highest mode of preparation in their study area.

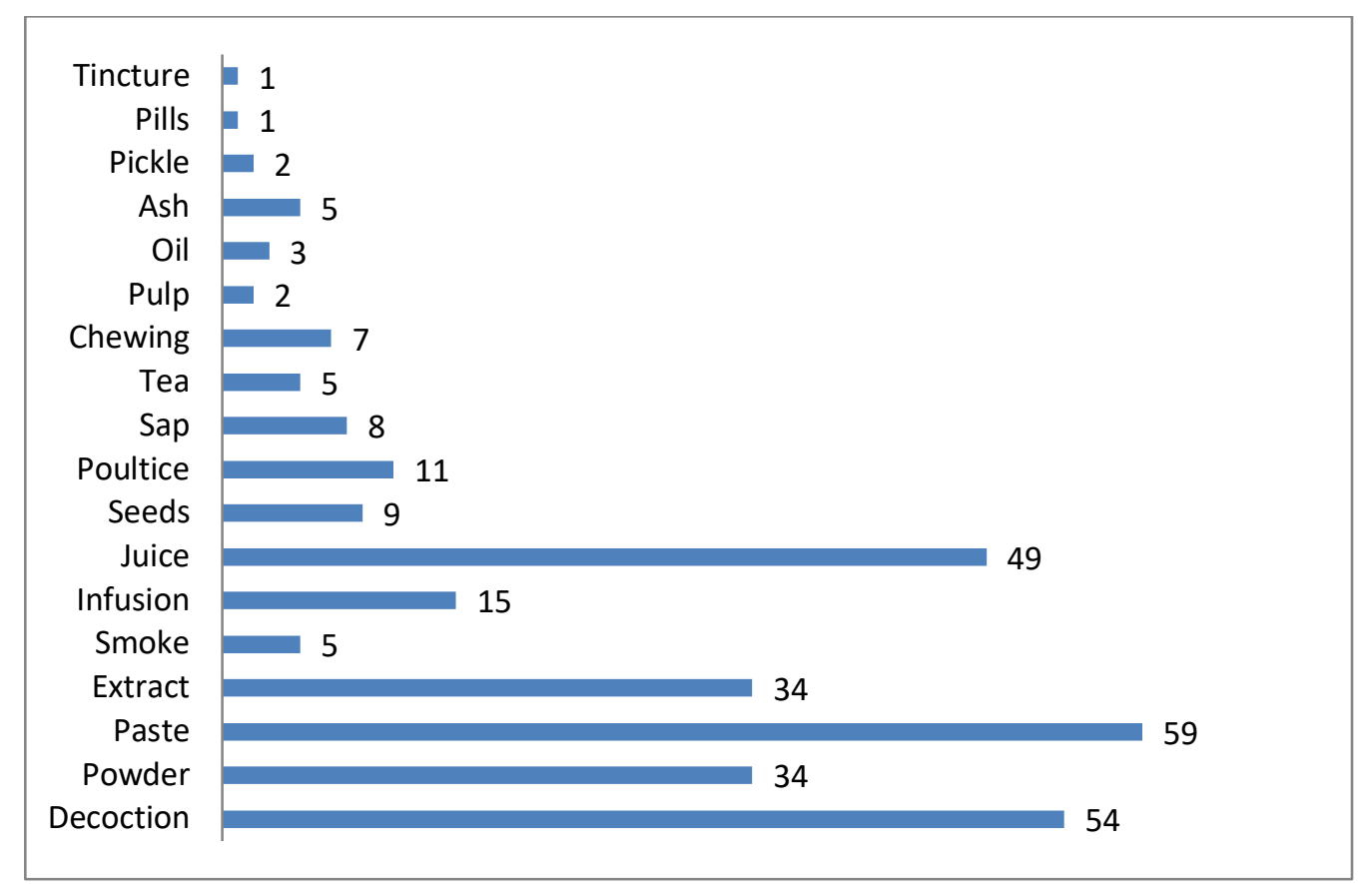

Figure 4. Mode of preparation used in present study area

\section{Use Value (UV)}

The use value (UV) index was applied to explore the ethno-botanical uses with respect to the previously reported medicinal plant species. In our study the calculated range of UV index was from $0.2-0.96$. The highest UV was reported for Cuscuta reflexa Roxb (0.96), followed by Melia azedarach L. (0.95), Ficus benghalensis L. (0.94), Tinospora sinensis (Lour.) Merr. and Withania somnifera (L.) Dunal (0.93 each) and Achyranthes aspera L. (0.91) respectively (Table 2). The high use reports of species indicate a strong relationship and dependence of local people on indigenous flora to treat various diseases and livelihood (Kayani et al. 2014).

\section{Relative Frequency of Citation (RFC)}

In present study, RFC value of reported plant species ranged from 0.03 to 0.55 . The RFC highest value was calculated for Ficus benghalensis L. (0.55), followed by Achyranthes aspera L. and Viola canescens Wall. (0.53 each), Mallotus philippensis (Lam.) Müll. Arg. (0.51) and Ficus semicordata Buch.-Ham. ex Sm. (0.48). The high RFC value of these medicinal plant species indicates that local people of study area use these plant species to cure various ailments and have a close association between local people and these plant species. The high RFC plant species were famous among local inhabitants of study area (Table 1). RFC indicates the importance of plant species with respect to information provided by local informants about use of plant species (Vitalini et al. 2013). Plant species having high RFC and UV should be exposed for further investigation such as pharmacological evaluation and phytochemical screening for commercial drug preparation (Mukherjee et al. 2012).

\section{Informant Consensus Factor (ICF)}

The ICF value was calculated against each disease category based on use reports, and it ranged from 0.21 to 0.68. The diseases were classified into 17 different categories (Table 2). The high range of ICF values recorded in study area confirms the higher dependability of local people on native flora especially for jaundice and hepatitis, cancer 
and tumors, circulatory system disorders, nervous disorders whereas low ICF value for cardiac problems reflected lesser consistency of informer's knowledge (Lin \& Mvelase 2002). Often few species have high use value and are only used for one disease category, reflecting high value of ICF (Madikizela et al. 2014), whereas the treatment of a particular disease category is conducted with high number of plant species confirms disagreement among the informants, reflecting a low value of ICF.

\section{Fidelity Level (FL)}

Plants having higher fidelity level (FL) value were mostly utilized on constant basis than those plants which had lower FL value. Species with high FL indicates their widespread use to treat a particular dominant disease of the area. In this study, the species having the highest FL value was Viola canescens Wall. (97.1\%) used in fever, flu, cough, constipation, and jaundice (Farooq et al. 2019) followed by Withania somnifera (L.) Dunal. (96.6\%) used in sexual problems, narcotics, ulcer, swellings, and joints pain, Phyllanthus emblica L. (96.4\%) used in diabetes, high blood pressure, diarrhea, and dysentery, Cuscuta reflexa Roxb. (96.0\%) used in fever, cough, blood purification, jaundice, skin problems, Melia azedarach L. (95.8) used in blood purification, malaria, skin disorders, febrifuge, Ficus auriculata Lour. (94.1\%) used in high blood pressure, diabetes, jaundice, wounds, diarrhea, dysentery, vomiting, cholera, and mumps, Cathranthes roseus (L.) G. Don. (88.4\%) used in diabetes, wounds, piles, high blood pressure, diarrhea, dysentery and anticancer, Carissa spinarum L. (87.5\%) used in asthma, jaundice, kidney pain, cardiac problems, and liver disorders, Ziziphus oxyphylla Edgew. (85.7\%) used in jaundice, diabetes, liver problems, allergy, and intestinal worms, Butea monosperma Taub. (82.3\%) used in diarrhea, dysentery, ulcer, swellings, cold cough, boils, pimples, wound and abortion pain, Bauhinia variegata L. (78.9\%) used in diarrhea, dysentery, skin problems, piles, and ulcer, Achyranthes aspera L. (66.6\%) used in rheumatic, diabetes, cough, asthma, skin problems and toothache and Barleria cristata L. (56.5\%) used in anemia, tooth pain, inflammation, diabetes, and fever. Plants having high value of Fidelity Level are recognized as model plants and hence can be used in further ethnopharmacological research (Hassan-Abdallah et al. 2013), (Table 3)

\section{Multipurpose Species}

In present study, out of 100 medicinal plant species, 30 species were used for other than medicinal purposes like vegetable, fruit, fodder, fuel, furniture, making agriculture tools, house thatching, walking sticks, hedge and fencing, construction, and handicrafts (Table 4). The number of uses varies from 2 to 7 . The highest number of use plant is Dodonaea viscosa (L.) Jacq. that is use as fodder, fuel, hedge, and fencing, making agriculture tools, house thatching, walking sticks and handicrafts. The most common plant part use is wood and mostly plant species used as fuel (22\%) (Table 8). Similarly, Ajaib et al. (2010) conducted ethnobotanical study of useful shrubs of District Kotli, Azad Kashmir in which plant species used as fuel, shelter, fodder, and agriculture tools.

\section{Direct Matrix Ranking of Multipurpose Species (DMRMS)}

In direct matrix ranking (DMR) (Table 5) of other than medicinal values of plants Dodonaea viscosa (L.) Jacq. ranked $1^{\text {st }}$ according to information provided by 10 informants of study area, followed by Pinus roxburghii Sarg. ( $\left.2^{\text {nd }}\right)$, Morus nigra L. $\left(3^{\text {rd }}\right)$, Olea ferruginea Wall. ex Aitch. $\left(4^{\text {th }}\right)$, Acacia modesta Wall. $\left(5^{\text {th }}\right)$, Grewia optiva J.R.Drumm. ex Burret $\left(6^{\text {th }}\right)$, Carissa spinarum L. $\left(7^{\text {th }}\right)$, Ficus auriculata Lour. $\left(8^{\text {th }}\right)$, Dalbergia sissoo DC. $\left(9^{\text {th }}\right)$, Bombax ceiba L. $\left(10^{\text {th }}\right)$. According to Khan et al., (2012) Morus nigra L., Ficus auriculata Lour. And Olea ferruginea Wall. ex Aitch., Morus nigra L., Grewia optiva J.R. Drumm. ex Burret were used as fruit and fodder respectively like our study. Some plant species in our study were used as muswak to clean teeth such as Dodonaea viscosa (L.) Jacq., Olea ferruginea Wall. ex Aitch. Zanthoxylum armatum DC. and Acacia modesta Wall. Similar study conducted by Khan et al. (2011) ethnobotany of some useful plants of Poonch valley Azad Kashmir in which Olea ferruginea Wall. ex Aitch., Acacia modesta Wall. and Zanthoxylum armatum DC were used as muswak to clean teeth. Khan et al. (2010) described that Dodonaea viscosa (L.) Jacq.used as wound healing, astringent, house thatching, fuel wood, fencing, toothbrushes and house cleaning brushes like our findings. Pinus roxburghii Sarg. used as timber, fuel wood, house building and medicinal purposes (Khan et al. 2010). Morus nigra L. used as fodder, tonic, cough, throat irritation, making agriculture tools, furniture, and fuel (Ajaib \& Khan 2014) like our study. 
Table 2. Informant Consensus Factor (ICF) of most common disease categories

\begin{tabular}{|c|c|c|c|c|c|c|}
\hline Use categories & Uses under each category & $\mathbf{N t}$ & Nur & Nur-Nt & Nur-1 & ICF \\
\hline Respiratory disorders & Cough, asthma, pneumonia, cold, flu & 42 & 54 & 12 & 53 & 0.22 \\
\hline Cardiovascular disorders & cardiac problems & 16 & 20 & 4 & 19 & 0.21 \\
\hline Digestive disorders & $\begin{array}{l}\text { Stomachic, digestant, dysentery, diarrhea, laxative, Constipation, dyspepsia, vomiting, cholera, nausea, } \\
\text { appetizer }\end{array}$ & 41 & 57 & 16 & 56 & 0.28 \\
\hline Skin problems & $\begin{array}{l}\text { Skin irritation, boils, abscess, pimples, burns, inflammation, wound, lesions, sores, itches, warts, } \\
\text { scabies, cracked heals }\end{array}$ & 36 & 48 & 12 & 47 & 0.25 \\
\hline Sexual problems & Gonorrhea, Spermatorrhoea, premature ejaculation, sexual desire & 14 & 18 & 4 & 17 & 0.23 \\
\hline Female problems & $\begin{array}{l}\text { Menstrual disorder, regularize menstrual cycle, leucorrhea, menstruation bleeding, increase milk } \\
\text { secretion, abortion, labor pain, gynecological disorder }\end{array}$ & 11 & 20 & 9 & 19 & 0.47 \\
\hline Bites & Snake bites, scorpion bites, dog bites & 24 & 38 & 14 & 37 & 0.37 \\
\hline Malaria and Fever & Malaria, fever, Dengue fever, typhoid fever & 20 & 29 & 9 & 28 & 0.32 \\
\hline Cancer and tumors & Cancer and tumors & 4 & 12 & 8 & 12 & 0.66 \\
\hline Diabetes & Diabetes & 26 & 43 & 17 & 42 & 0.40 \\
\hline Jaundice and Hepatitis & Jaundice and Hepatitis & 6 & 17 & 11 & 16 & 0.68 \\
\hline Pain & Joints pain, waist pain, toothache, stomachache, headache, backache, body pain, arthritis & 32 & 51 & 19 & 50 & 0.38 \\
\hline $\begin{array}{l}\text { Circulatory system } \\
\text { disorders }\end{array}$ & Blood purifier, Blood cholesterol level, anemia, High blood pressure, blood poisoning & 12 & 35 & 23 & 34 & 0.67 \\
\hline Ulcers & Mouth ulcer, kidney ulcer, bladder ulcer & 14 & 27 & 13 & 26 & 0.5 \\
\hline Gastric problems & Carminative, anthelminthic, anthelmintics & 16 & 32 & 16 & 31 & 0.51 \\
\hline Nervous disorders & Narcotics, painkillers, insomnia, sedative, & 3 & 6 & 3 & 5 & 0.6 \\
\hline General disorders & $\begin{array}{l}\text { Piles, tonic, stimulant, anti-fertility, kidney problems, eye problems, stop bleeding, antiseptic, } \\
\text { antibacterial, expectorant, obesity, mumps, tetanus, liver disorders, epilepsy, Tuberculosis. }\end{array}$ & 34 & 47 & 13 & 46 & 0.28 \\
\hline
\end{tabular}


Table 3: Fidelity level (FL) values of commonly reported medicinal plants of study area

\begin{tabular}{|c|c|c|c|c|c|}
\hline Scientific name & Local name & Disease categories & Ip & Iu & FL (\%) \\
\hline Achyranthes aspera L. & PuthKanda & $\begin{array}{l}\text { Rheumatic, diabetic, cough, asthma, skin irritation, joints pains, piles, boil, abscess, stomach pain, digestant, } \\
\text { pneumonia, toothache, anti-fertility. }\end{array}$ & 12 & 18 & 66.6 \\
\hline Barleria cristata L. & Kali Barenker & $\begin{array}{l}\text { Anemia, tooth pain, inflammation, wound, snake bite, fever, diabetes, tuberculosis, cold, flu, diaphoretic, } \\
\text { antibacterial and expectorant. }\end{array}$ & 13 & 23 & 56.5 \\
\hline Bauhinia variegata $\mathrm{L}$. & Kalyar & $\begin{array}{l}\text { Diarrhea, dysentery, wound, skin disorders, ulcers, worms, dyspepsia, piles, regularize menstrual cycle, } \\
\text { carminative, obesity, laxative, anthelmintics, tonic. }\end{array}$ & 15 & 19 & 78.9 \\
\hline $\begin{array}{l}\text { Butea monosperma } \\
\text { Taub. }\end{array}$ & Chachra & $\begin{array}{l}\text { Diarrhea, dysentery, ulcers, swellings, ringworm, cold, cough, boils, pimples, wounds, leucorrhea, control } \\
\text { bleeding, abortion pain, anthelminthic. }\end{array}$ & 14 & 17 & 82.3 \\
\hline Carissa spinarum $\mathrm{L}$. & Granda & $\begin{array}{l}\text { Asthma, jaundice, kidney pain, cardiac problems, stimulant, urine blockage, urinary bladder, remittent fever, } \\
\text { liver disorders, blood deficiencies, wounds, sores, stomachic, purgative }\end{array}$ & 7 & 8 & 87.5 \\
\hline $\begin{array}{l}\text { Catharanthus roseus } \\
\text { (L.) G. Don. }\end{array}$ & Sada bahar & $\begin{array}{l}\text { Diabetes, wounds, dysmenorrhea, piles, nasal and mouth bleeding, high blood pressure, irregular menstruation, } \\
\text { dysentery, diarrhea, anticancer. }\end{array}$ & 23 & 26 & 88.4 \\
\hline Cissampelos pareira L. & Battal Bel & $\begin{array}{l}\text { Relief tooth pain, leucorrhea, snake bite, carminative, astringent, diuretic, expectorant, sedative, febrifuge, } \\
\text { oblivion, gastrointestinal problems, fever, cold, typhoid fever, anti-conjunctivitis. }\end{array}$ & 10 & 19 & 52.6 \\
\hline Cuscuta reflexa Roxb. & Neela tari & $\begin{array}{l}\text { Astringent, purgative, anthelmintic, baldness, wound, anti-lice, hair tonic, diarrhea, itches, sores, gout, joint } \\
\text { pains, skin itching, jaundice, skin diseases, cough, blood purification, fever, menstruation bleeding. }\end{array}$ & 24 & 25 & 96.0 \\
\hline Ficus auriculata Lour. & Tossy & High blood pressure, cuts, wounds, diarrhea, dysentery, cholera, mumps, vomiting, diabetes, jaundice. & 16 & 17 & 94.1 \\
\hline Gloriosa superba L. & Charkiara & $\begin{array}{l}\text { Stimulant, abortifacient, anthelmintic, leprosy, antidote to snake poison, earache, toothache, asthma, wounds, } \\
\text { pimples, malaria, abdominal problems, reduce abortion. }\end{array}$ & 6 & 13 & 46.1 \\
\hline Melia azedarach L. & Dahrek & $\begin{array}{l}\text { Blood purification, tonic for stomach and liver, spleen enlargement, ascariasis, dandruff, eczema, ringworms, } \\
\text { skin itching, malaria, antiseptic and febrifuge. }\end{array}$ & 23 & 24 & 95.8 \\
\hline Phyllanthes emblica L. & Amla & Diabetes, high blood pressure, cooling, astringent, laxative, diuretic, diarrhea, dysentery, and eye problems. & 27 & 28 & 96.4 \\
\hline Viola canescens Wall. & Banfsha & Fever, flu, cough, constipation, laxative, skin abrasions, jaundice. & 34 & 35 & 97.1 \\
\hline $\begin{array}{l}\text { Withania somnifera } \\
\text { (L.) Dunal. }\end{array}$ & Ashgand & $\begin{array}{l}\text { Diuretic, tonic and aphrodisiac, sexual problems and promotes urination, spermatorrhoea, narcotics, ulcer, } \\
\text { swelling, diuretic, joints pains, skin sore, inflammations, insomnia, stress, and anxiety. }\end{array}$ & 29 & 30 & 96.6 \\
\hline $\begin{array}{l}\text { Ziziphus oxyphylla } \\
\text { Edgew }\end{array}$ & Mimyaon & Jaundice, diabetes and liver problems, hypertension and gas troubles, allergy, intestinal worms. & 12 & 14 & 85.7 \\
\hline
\end{tabular}


Table 4. Plant species having multipurpose used other than medicinal values

\begin{tabular}{|c|c|c|c|c|c|c|c|c|c|c|c|c|c|c|}
\hline \multirow[t]{2}{*}{ Plant species } & \multirow[t]{2}{*}{ Local name } & \multirow[t]{2}{*}{ Part used } & \multicolumn{11}{|c|}{ Cultural uses } & \multirow{2}{*}{$\begin{array}{l}\text { No. of } \\
\text { uses }\end{array}$} \\
\hline & & & $\mathbf{V}$ & $\mathbf{F}$ & Fo & $\mathbf{F u}$ & Fn & $\mathbf{C}$ & HF & AT & HT & $\mathbf{S}$ & HC & \\
\hline Acacia modesta Wall. & Phalai & $L, W$ & 0 & 0 & 1 & 1 & 1 & 0 & 0 & 0 & 0 & 0 & 0 & 3 \\
\hline Albizzia lebbeck (L.) Bth. & Shirin & L, W & 0 & 0 & 1 & 1 & 0 & 0 & 0 & 0 & 0 & 0 & 0 & 2 \\
\hline Bombax ceiba L. & Simbal & W, F & 0 & 0 & 0 & 0 & 1 & 1 & 0 & 0 & 0 & 1 & 1 & 4 \\
\hline Bauhinia variegata $\mathrm{L}$. & Kalyar & $\mathrm{L}, \mathrm{Fd}$ & 1 & 0 & 1 & 1 & 0 & 0 & 0 & 0 & 0 & 0 & 0 & 3 \\
\hline Buddleja asiatica Lour. & Bhati & $\mathrm{W}$ & 0 & 0 & 0 & 1 & 0 & 0 & 0 & 0 & 0 & 1 & 0 & 2 \\
\hline Carissa spinarum L. & Granda & Wh, L, F & 0 & 1 & 1 & 0 & 0 & 0 & 1 & 0 & 0 & 0 & 0 & 3 \\
\hline Dalbergia sissoo DC. & Tali & W & 0 & 0 & 0 & 1 & 1 & 1 & 0 & 1 & 0 & 0 & 0 & 4 \\
\hline Dodonaea viscosa (L.) Jacq. & Sanatha & W & 0 & 0 & 1 & 1 & 0 & 0 & 1 & 1 & 1 & 1 & 1 & 7 \\
\hline Ficus auriculata Lour. & Tossy & $L, F, W$ & 1 & 1 & 1 & 1 & 0 & 0 & 0 & 0 & 0 & 0 & 0 & 4 \\
\hline Ficus benghalensis $\mathrm{L}$. & Bohr & $\mathrm{L}, \mathrm{F}$ & 0 & 1 & 1 & 0 & 0 & 0 & 0 & 1 & 0 & 0 & 1 & 4 \\
\hline Ficus palmata Forssk. & Phagwar & $L, F$ & 0 & 1 & 1 & 1 & 0 & 0 & 0 & 0 & 0 & 0 & 0 & 3 \\
\hline Ficus racemosa $\mathrm{L}$. & Rumbal & $L, F$ & 1 & 1 & 1 & 1 & 0 & 0 & 0 & 0 & 0 & 0 & 0 & 4 \\
\hline Ficus religiosa $\mathrm{L}$. & Peepal & $\mathrm{L}$ & 0 & 0 & 1 & 1 & 0 & 0 & 0 & 0 & 0 & 0 & 0 & 2 \\
\hline Ficus semicordata Buch.-Ham. ex Sm. & Panjal & $L, F$ & 0 & 1 & 1 & 1 & 0 & 0 & 0 & 0 & 0 & 0 & 0 & 3 \\
\hline Flacourtia indica (Burm. f.) Merr. & Koko & $\mathrm{L}, \mathrm{F}$ & 0 & 1 & 1 & 0 & 0 & 0 & 0 & 0 & 0 & 0 & 0 & 2 \\
\hline Grewia optiva J. R. Drumm. & Dahman & $L, F, W$ & 0 & 1 & 1 & 1 & 0 & 0 & 0 & 0 & 0 & 0 & 1 & 4 \\
\hline Leucaena leucocephala (Lam.) de Wit. & Babul & $\mathrm{L}, \mathrm{W}$ & 0 & 0 & 1 & 1 & 0 & 0 & 0 & 0 & 0 & 0 & 0 & 2 \\
\hline Mallotus philippensis (Lam.) Mull. Arg. & Kamela & W & 0 & 0 & 0 & 1 & 0 & 0 & 0 & 1 & 0 & 1 & 0 & 3 \\
\hline Melia azedarach $\mathrm{L}$. & Dahrek & $\mathrm{L}, \mathrm{W}$ & 0 & 0 & 1 & 1 & 1 & 0 & 0 & 0 & 0 & 0 & 0 & 3 \\
\hline Morus nigra L. & Kala toot & $L, F, W$ & 0 & 1 & 1 & 1 & 0 & 0 & 0 & 0 & 0 & 0 & 1 & 4 \\
\hline Olea ferruginea Wall. ex Aitch. & Kao & W & 0 & 0 & 0 & 0 & 0 & 0 & 0 & 1 & 0 & 1 & 0 & 2 \\
\hline Pinus roxburghii Sarg. & Cheer & $\mathrm{W}$ & 0 & 0 & 0 & 1 & 1 & 1 & 0 & 0 & 1 & 0 & 0 & 4 \\
\hline Rubus ellipticus Sm. & Akhre & Wh, F & 0 & 1 & 0 & 0 & 0 & 0 & 1 & 0 & 0 & 0 & 0 & 2 \\
\hline Saccharum benghalense Retz. & Saroot & $\mathrm{Cu}$ & 0 & 0 & 0 & 0 & 0 & 0 & 0 & 0 & 1 & 0 & 1 & 2 \\
\hline $\begin{array}{l}\text { Wendlandia heynei (Schult.) Santapau \& } \\
\text { Merchant }\end{array}$ & Okan & W & 0 & 0 & 0 & 1 & 0 & 1 & 0 & 0 & 1 & 0 & 0 & 3 \\
\hline Zanthoxylum armatum DC. & Timber & $\mathrm{F}, \mathrm{W}$ & 0 & 1 & 0 & 0 & 0 & 0 & 0 & 0 & 0 & 1 & 0 & 2 \\
\hline Ziziphus jujuba Mill. & Jahnd & $\mathrm{L}, \mathrm{F}$ & 0 & 1 & 1 & 0 & 0 & 0 & 0 & 0 & 0 & 0 & 0 & 2 \\
\hline Total & & & 3 & 12 & 17 & 18 & 5 & 4 & 3 & 5 & 4 & 6 & 6 & \\
\hline
\end{tabular}

Cultural uses: $V$ vegetable, F fruit, Fo fodder, Fu fuel, Fn furniture, C construction, HFhedges and fencing, $A T$ agricultural tools, $H T$ house thatching, $S$ walking sticks, $H C$ handicraft.

Part used: $\angle$ leaves, $W$ wood, $F d$ floral bud, $F$ fruit, $W h$ whole plant, $C u$ culms. 
Ethnobotany Research and Applications

Table 5. DMR of dominant woody plant species having other than medicinal values (information collected from 10 informants)

\begin{tabular}{|c|c|c|c|c|c|c|c|c|c|c|}
\hline Uses & $\begin{array}{l}\text { Dodoaeae } \\
\text { viscosa }\end{array}$ & $\begin{array}{l}\text { Pinus } \\
\text { roxburghii }\end{array}$ & $\begin{array}{l}\text { Dalbergea } \\
\text { sissoo }\end{array}$ & $\begin{array}{l}\text { Carissa } \\
\text { spinarum }\end{array}$ & $\begin{array}{l}\text { Ficus } \\
\text { auriculata }\end{array}$ & $\begin{array}{l}\text { Grewia } \\
\text { optiva }\end{array}$ & $\begin{array}{l}\text { Olea } \\
\text { ferruginea }\end{array}$ & $\begin{array}{l}\text { Acacia } \\
\text { modesta }\end{array}$ & $\begin{array}{l}\text { Bombax } \\
\text { ceiba }\end{array}$ & $\begin{array}{l}\text { Morus. } \\
\text { nigra }\end{array}$ \\
\hline $\mathbf{V}$ & 0 & 0 & 0 & 0 & 6 & 0 & 0 & 0 & 0 & 0 \\
\hline $\mathbf{F}$ & 0 & 8 & 0 & 10 & 10 & 4 & 0 & 0 & 0 & 10 \\
\hline Fo & 10 & 0 & 0 & 10 & 8 & 10 & 8 & 10 & 0 & 10 \\
\hline $\mathbf{F u}$ & 10 & 10 & 10 & 0 & 6 & 10 & 7 & 10 & 3 & 10 \\
\hline Fn & 0 & 9 & 10 & 0 & 0 & 0 & 0 & 10 & 0 & 0 \\
\hline AT & 10 & 0 & 0 & 0 & 0 & 0 & 10 & 0 & 0 & 0 \\
\hline HT & 8 & 7 & 0 & 0 & 0 & 0 & 0 & 0 & 0 & 0 \\
\hline C & 0 & 8 & 0 & 0 & 0 & 0 & 0 & 0 & 0 & 0 \\
\hline HF & 6 & 0 & 0 & 5 & 0 & 0 & 0 & 0 & 0 & 0 \\
\hline WS & 7 & 0 & 0 & 0 & 0 & 0 & 9 & 0 & 2 & 0 \\
\hline $\mathrm{HC}$ & 6 & 0 & 3 & 0 & 0 & 5 & 0 & 0 & 6 & 8 \\
\hline Total & 57 & 42 & 23 & 25 & 24 & 29 & 34 & 30 & 11 & 38 \\
\hline Rank & $1^{\text {st }}$ & $2^{\text {nd }}$ & $9^{\text {th }}$ & $7^{\text {th }}$ & $8^{\text {th }}$ & $6^{\text {th }}$ & $4^{\text {th }}$ & $5^{\text {th }}$ & $10^{\text {th }}$ & $3^{\text {rd }}$ \\
\hline
\end{tabular}




\section{Conclusion}

It was concluded that the region of Tehsil Charhoi, District Kotli, AJ\&K is rich source of wild medicinal plant species. The aim of the present research to explored ethnobotanical indigenous knowledge of present area. This research established the traditional knowledge of medicinal plants used in Tehsil Charhoi District Kotli AJ\&K was low or facing erosion. There is, therefore, an urgent need to document this information, as it is rapidly disappearing due to influence of western medicines and other reasons and other reasons including socio-cultural issues and our exploitation coupled with rapid deforestation. The main reason of deforestation is fire. The fire force the other wild animals too migrate to other allied areas. Therefore, there is need to aware local people to stop happening of fire. Due to all these reasons, there is need to collect ethnobotanical data and published to develop a database of medicinal plants for future research and potential development of new drugs. This publication helps to save ethnobotanical data of this area for future generations. There is need to create awareness among the local people for the collection of these medicinal plants and control deforestation.

\section{Declarations}

List of abbreviations: Not applicable.

Ethical approval and consent to participate: This study was approved by the Departmental Research and Review Committee (DRRC) and Ethics Committee of Advanced Studies and Research Board (ASRB), University of Gujrat, Pakistan.

Consent for publication: Not applicable

Conflict of interests: The authors declare that they have no conflict of interests.

Funding: This research work did not receive any grant from funding agencies in the public, commercial, or notfor-profit sectors.

Authors' contributions: $\mathrm{UA}$, KH designing of the study and proposed the study area; KH, MA and II participated in the collection of field data and identification of plant samples. MM and JZ identified the plant species reference herbarium and flora. JK analysed the data and wrote the initial draft of the manuscript. All the authors participated in writing and giving feedback on the manuscript and approved the final version of the manuscript.

\section{Literature cited}

Abbas W, Hussain W, Hussain W, Badshah L, Hussain K, Pieroni A. 2020. Traditional wild vegetables gathered by four religious groups in Kurram District, Khyber Pakhtunkhwa, North-West Pakistan. Genetic Resources and Crop Evolution 67(6):1521-1536.

Ahmad KS, Hamid A, Nawaz F, Hameed M, Ahmad F, Deng J, Akhtar N, Wazarat A, Mahroof S. 2017. Ethnopharmacological studies of indigenous plants in Kel village, Neelum Valley, Azad Kashmir, Pakistan. Journal of Ethnobiology and Ethnomedicine 13(1):1-6.

Ahmad KS, Kayani WK, Hameed M, Ahmad F, Nawaz T. 2012. Floristic diversity and ethnobotany of senhsa, district Kotli, Azad Jammu \& Kashmir (Pakistan). Pakistan Journal of Botany 44:195-201.

Ahmad M, Khan MP, Mukhtar A, Zafar M, Sultana S, Jahan S. 2016. Ethnopharmacological survey on medicinal plants used in herbal drinks among the traditional communities of Pakistan. Journal of Ethnopharmacology 184:154-186.

Ajaib M, Abid A, Ishtiaq M. 2016. Ethnobotanical studies of wild plant resources of Puna Hills, District Bhimber, AJK. FUUAST Journal of Biology 6(2):257-264.

Ajaib M, Khan Z, Khan NA, Wahab M. 2010. Ethnobotanical studies on useful shrubs of district Kotli, Azad Jammu \& Kashmir, Pakistan. Pakistan Journal of Botany 42(3):1407-1415.

Ajaib M, Khan Z. 2014. Ethnobotanical studies of useful trees of District Kotli, Azad Jammu and Kashmir. Biologia Pakistan 60(13):63-71.

Ajaib MU, Anjum MU, Malik NZ, Sidiqui MF. 2015. Ethnobotanical study of some plants of Darguti, tehsil Khuiratta, Azad Jammu and Kashmir. International Journal of Biological Research 3(9):101-107.

Akinyemi KO, Oluwa OK, Omomigbehin EO. 2006. Antimicrobial activity of crude extracts of three medicinal plants used in south-west Nigerian folk medicine on some food borne bacterial pathogens. African Journal of Traditional, Complementary and Alternative Medicines 3(4):13-22.

Altaf R, Bhatti KH, Mirza SA, Ajaib M, Ishtiaq M. 2019. Ethnomedicinal Study of Tehsil Wazirabad Gujranwala Punjab Pakistan. Pakistan Journal of Science 71(4):248-260. 
Amiri MS, Joharchi MR. 2013. Ethnobotanical investigation of traditional medicinal plants commercialized in the markets of Mashhad, Iran. Avicenna Journal of Phytomedicine 3(3):243-254.

Amjad MS, Arshad M, Saboor A, Page S, Chaudhari SK. 2017. Ethnobotanical profiling of the medicinal flora of Kotli, Azad Jammu and Kashmir, Pakistan: Empirical reflections on multinomial logit specifications. Asian Pacific Journal of Tropical Medicine 10(5):503-514.

Amjad MS, Arshad M. 2014.Ethnobotanical inventory and medicinal uses of some important woody plant species of Kotli, Azad Kashmir, Pakistan. Asian Pacific Journal of Tropical Biomedicine 4(12):952-958.

Amjad MS, Qaeem MF, Ahmad I, Khan SU, Chaudhari SK, Zahid Malik N, Shaheen H, Khan AM. 2017. Descriptive study of plant resources in the context of the ethnomedicinal relevance of indigenous flora: A case study from Toli Peer National Park, Azad Jammu and Kashmir, Pakistan. PloS one 12(2):871-896.

Amjad MS, Zahoor U, Bussmann RW, Altaf M, Gardazi SM, Abbasi AM. 2020. Ethnobotanical survey of the medicinal flora of Harighal, Azad Jammu \& Kashmir, Pakistan. Journal of Ethnobiology and Ethnomedicine 16(1):1-28.

Amjad MS. 2015. Ethnobotanical profiling and floristic diversity of Bana Valley, Kotli (Azad Jammu and Kashmir), Pakistan. Asian Pacific Journal of Tropical Biomedicine 5(4):292-299.

Anonymous. 2020. Annual rainfall, relative humidity and temperature of Tehsil Charhoi, District Kotli, Azad Jammu \& Kashmir 2018-2020. Pakistan Metrological Department Jail Road Lahore, Pakistan.

Bano A, Ahmad M, Hadda TB, Saboor A, Sultana S, Zafar M, Khan MP, Arshad M, Ashraf MA. 2014. Quantitative ethnomedicinal study of plants used in the skardu valley at high altitude of Karakoram-Himalayan range, Pakistan. Journal of Ethnobiology and Ethnomedicine 10(1):1-8.

Calixto JB. 2005. Twenty-five years of research on medicinal plants in Latin America: a personal view. Journal of Ethnopharmacology 100(1-2):131-134.

Calzada F, Bautista E. 2020. Plants used for the treatment of diarrhoea from Mexican flora with amoebicidal and giadicidal activity, and their phytochemical constituents. Journal of Ethnopharmacology 253:112-176.

Ch MI, Ahmed F, Maqbool M, Hussain T. 2013. Ethnomedicinal inventory of flora of maradori valley, district forward Khahuta, Azad Kashmir, Pakistan. American Journal of Research Communication 1(6):239-261.

Chitme HR, Chandra R, Kaushik S. 2004. Studies on anti-diarrhoeal activity of Calotropis gigantea R. Br. in experimental animals..Journal of Pharmaceutical Sciences7(1):70-85.

Farooq A, Amjad MS, Ahmad K, Altaf M, Umair M, Abbasi AM. 2019. Ethnomedicinal knowledge of the rural communities of Dhirkot, Azad Jammu and Kashmir, Pakistan. Journal of Ethnobiology and Ethnomedicine 15(1):130.

Faruque MO, Uddin SB, Barlow JW, Hu S, Dong S, Cai Q, Li X, Hu X. 2018. Quantitative ethnobotany of medicinal plants used by indigenous communities in the Bandarban District of Bangladesh. Frontiers in pharmacology 9(1):4056.

Ghorbani A. 2005. Studies on pharmaceutical ethnobotany in the region of Turkmen Sahra, north of Iran:(Part 1): General results. Journal of Ethnopharmacology 102(1):58-68.

Giday M, Asfaw Z, Woldu Z. 2009. Medicinal plants of the Meinit ethnic group of Ethiopia: an ethnobotanical study. Journal of Ethnopharmacology 124(3):513-521.

Hassan-Abdallah A, Merito A, Hassan S, Aboubaker D, Djama M, Asfaw Z, Kelbessa E. 2013. Medicinal plants and their uses by the people in the Region of Randa, Djibouti. Journal of ethnopharmacology 148(2):701-713.

Ishtiaq M, Mahmood A, Maqbool M. 2015. Indigenous knowledge of medicinal plants from Sudhanoti district (AJK), Pakistan. Journal of Ethnopharmacology 168:201-207.

Ishtiaq M, Maqbool M, Ajaib M, Ahmed M, Hussain I, Khanam H, Mushtaq W, Hussain T, Azam S, Hayat Bhatti K, Ghani A. 2021. Ethnomedicinal and folklore inventory of wild plants used by rural communities of valley Samahni, District Bhimber Azad Jammu and Kashmir, Pakistan. PloS one 16(1):243-251. 
Ishtiaq M, Maqbool M, Hussain T, Shah A. 2013. Role of indigenous knowledge in biodiversity conservation of an area: a case study on tree ethnobotany of Soona Valley, District Bhimber Azad Kashmir, Pakistan. Pakistan Journal of Botany 45(157):245-56.

Kayani S, Ahmad M, Zafar M, Sultana S, Khan MP, Ashraf MA, Hussain J, Yaseen G. 2014. Ethnobotanical uses of medicinal plants for respiratory disorders among the inhabitants of Gallies-Abbottabad, Northern Pakistan. Journal of Ethnopharmacology 156:47-60.

Khan MA, Khan MA, Hussain M, Ghulam GM. 2010. An ethnobotanical inventory of himalayan region poonch valley azad kashmir (Pakistan). Ethnobotany Research and Applications 8:107-123.

Khan MA, Khan MA, Mujtaba G, Hussain M. 2012.Ethnobotanical study about medicinal plants of Poonch valley Azad Kashmir.Journal of Animal and Plant Sciences 22(2):493-500.

Khan MA, Khan SA, Qureshi MA, Ahmed G, Khan MA, Hussain M, Ghulam GM. 2011. Ethnobotany of some useful plants of Poonch Valley Azad Kashmir. Journal of Medicinal Plants Research 5(26):6140-6151.

Kritikar KR, Basu BD. 2001. Indian medicinal plants with illustrations, Dehradun: Oriental Enterprises 4(1):10-61.

Lin J, Puckree T, Mvelase TP. 2002. Anti-diarrhoeal evaluation of some medicinal plants used by Zulu traditional healers. Journal of Ethnopharmacology 79(1):53-56.

Madikizela B, Ndhlala AR, Finnie JF, Van Staden J. 2014.Antimycobacterial, anti-inflammatory and genotoxicity evaluation of plants used for the treatment of tuberculosis and related symptoms in South Africa. Journal of Ethnopharmacology 153(2):386-391.

Mahmood A, Mahmood A, Shaheen H, Qureshi RA, Sangi Y, Gilani SA. 2011. Ethnomedicinal survey of plants from district Bhimber Azad Jammu and Kashmir, Pakistan. Journal of Medicinal Plants Research 5(11):2348-2360.

Malik AY, Singh D. 2019. Ethnobotanical and ethnoveterinary importance of plants of scrub areas of Dachigam national park, Jammu and Kashmir, India.Asian Journal of Pharmaceutical Clinical Research 12(3):582-586.

Miller AG, Nyberg JA. 1995. Collecting herbarium vouchers. Royal Botanic Garden, Edinburgh EH3 5LR, UK, 561573.

Mukherjee PK, Nema NK, Venkatesh P, Debnath PK. 2012.Changing scenario for promotion and development of Ayurveda-way forward. Journal of Ethnopharmacology 143(2):424-434.

Qureshi RA, Gilani SA, Ashraf M. 2007. Ethnobotanical studies with special reference to plants phenology at Sudhan Gali and Ganga Chotti Hills (District Bagh, AK). Electronic Journal of Environmental, Agricultural and Food Chemistry 6(7):2207-2215.

Rao RN, Henry AN. 1997. The Ethnobotany of Eastern Ghats in Andhra Pradesh, India.Botanical Survey of India, Calcutta259(5):12-16.

Rashid A, Arshad M. 2002. Medicinal plant diversity, threat imposition and interaction of a mountain people community. In Proceeding of Workshop on Curriculum Development in Applied Ethnobotany.Published by the Ethnobotany Project, WWF Pakistan 3(1):84-90.

Rehman A, Hussain K, Nawaz K, Arshad N, Iqbal I, Ali SS, Nazeer A, Bashir Z, Jafar S, Arif U. 2020. Indigenous knowledge and medicinal significance of seasonal weeds of district Gujrat, Punjab, Pakistan. Ethnobotany Research and Applications 20(2):1-9.

Rodrigues E, Cassas F, Conde BE, Da Cruz C, Barretto EH, Dos Santos G, Figueira GM, Passero LF, Dos Santos MA, Gomes MA, Matta P. 2020. Participatory ethnobotany and conservation: a methodological case study conducted with quilombola communities in Brazil's Atlantic Forest. Journal of Ethnobiology and Ethnomedicine 16(1):1-12.

Saghir IA, Awan AA, Majid S. 2001. Ethnobotanical Studies of Chikar and its Allied Areas of District Muzaffarabad. Online Journal of Biological Sciences.1(12):1165-1170.

Shabir A, Naveed I, Uneeza J, Noor UA, Hina J, Farhat Y. 2017. Ethno botanical Wisdom of Inhabitant of Devi Galli Azad Kashmir. Biomedical Journal of Scientific \& Technical Research1(6):1618-1627.

Sulaiman SS, Khan S, Bussmann RW, Ali M, Hussain D, Hussain W. 2020. Quantitative Ethnobotanical Study of Indigenous Knowledge on Medicinal Plants Used by the Tribal Communities of Gokand Valley, District Buner, Khyber Pakhtunkhwa, Pakistan. Plants 9(8):1-10. 
Supiandi MI, Zubaidah S, Mahanal S, Julung H, Ege B. 2019. An ethnobotanical study of medicinal plants used by Dayak Desa from Tintau Menuah forest and Genik Luak Bukit Bang. Medicinal Plants-International Journal of Phytomedicines and Related Industries 11(3):292-306.

Tesfaye S, Belete A, Engidawork E, Gedif T, Asres K. 2020. Ethnobotanical study of medicinal plants used by traditional healers to treat cancer-like symptoms in eleven districts, Ethiopia. Evidence-Based Complementary and Alternative Medicine 46(4):506-522.

Umair M, Altaf M, Abbasi AM. 2017. An ethnobotanical survey of indigenous medicinal plants in Hafizabad district, Punjab-Pakistan. PloS one 12(6):1-22.

Vitalini S, Iriti M, Puricelli C, Ciuchi D, Segale A, Fico G. 2013. Traditional knowledge on medicinal and food plants used in Val San Giacomo (Sondrio, Italy) - An alpine ethnobotanical study. Journal of Ethnopharmacology 145(2):517-529.

Wagay NA, 2014. Medicinal flora and Ethno-botanical knowledge of Baramulla Tehsil in Jammu and Kashmir, India. International Journal of Advanced Biotechnology and Research, 5(3):539-546.

Wagh VV, Jain AK. 2020. Ethnopharmacological survey of plants used by the Bhil and Bhilala ethnic community in dermatological disorders in Western Madhya Pradesh, India. Journal of Herbal Medicine. 19:100-134.

Yeung AWK, Heinrich M, Kijjoa A, Tzvetkov NT, Atanasov AG. 2020. The ethnopharmacological literature: An analysis of the scientific landscape. Journal of Ethnopharmacology, 250:112-124. 